\title{
Spectral theory of fluctuations in time-average statistical mechanics of reversible and driven systems
}

\author{
Alessio Lapolla $\odot$, David Hartich, and Aljaž Godec $\odot^{*}$ \\ Mathematical bioPhysics group, Max Planck Institute for Biophysical Chemistry, Göttingen 37077, Germany
}

(Received 20 April 2020; accepted 25 August 2020; published 15 October 2020)

\begin{abstract}
We present a spectral-theoretic approach to time-average statistical mechanics for general, nonequilibrium initial conditions. We consider the statistics of bounded, local additive functionals of reversible as well as irreversible ergodic stochastic dynamics with continuous or discrete state-space. We derive exact results for the mean, fluctuations, and correlations of time-average observables from the eigenspectrum of the underlying generator of Fokker-Planck or master equation dynamics, and we discuss the results from a physical perspective. Feynman-Kac formulas are rederived using Itô calculus and combined with non-Hermitian perturbation theory. The emergence of the universal central limit law in a spectral representation is shown explicitly on largedeviation timescales. For reversible dynamics with equilibrated initial conditions, we derive a general upper bound to fluctuations of occupation measures in terms of an integral of the return probability. Simple, exactly solvable examples are analyzed to demonstrate how to apply the theory. As a biophysical example, we revisit the Berg-Purcell problem on the precision of concentration measurements by a single receptor. Our results are directly applicable to a diverse range of phenomena underpinned by time-average observables and additive functionals in physical, chemical, biological, and economical systems.
\end{abstract}

DOI: 10.1103/PhysRevResearch.2.043084

\section{INTRODUCTION}

Many experiments on soft and biological matter, such as single-particle tracking [1-4] and single-molecule spectroscopy [5-13], probe individual trajectories. It is typically not feasible to repeat these experiments enough times to allow for ensemble-averaging. It is, however, straightforward to analyze such data by means of time-averaging along individual realizations. However, except for (ergodically) long observations, time-averages inferred from individual trajectories are random with nontrivial statistics. This naturally leads to the study of statistical properties of time-averages which formally represent functionals of stochastic processes.

The study of functionals of stochastic processes has a long tradition in mathematics (see, e.g., [14-21]) and finance $[22,23]$. In physics, they were found to be relevant in the context of diffusion-controlled chemical reactions (e.g., [24-26]), transport in porous media [27], chemical inference [28-37], astrophysical observations [38], medical diagnostics [39], optical imaging [40], the study of growing surfaces [41], blinking of colloidal quantum dots [42,43], mesoscopic physics [44], climate [45] and computer science [46], and most recently in single-molecule spectroscopy [47-50] and diffusion studies [51], to name a few.

\footnotetext{
*agodec@mpibpc.mpg.de

Published by the American Physical Society under the terms of the Creative Commons Attribution 4.0 International license. Further distribution of this work must maintain attribution to the author(s) and the published article's title, journal citation, and DOI.
}

From a theoretical point of view, analytical results were obtained for the occupation time statistics for discrete-state Markov switching [47-50], for the local time at zero and occupation time above zero of a Brownian particle diffusing in a simple one-dimensional potential $[46,52,53]$, the occupation time inside a spherical domain of a Brownian particle moving in free space [51], and for a free, uniformly biased and harmonically bound particle undergoing subdiffusion [54,55]. Exact results were also obtained for occupation time statistics for a general class of Markov processes [56] and a discrete stationary non-Markovian sequence [57]. Large deviation functions for various nonlinear functionals of a class of Gaussian stationary Markov processes were studied in [45]. Numerous important results on functionals have also been obtained in the context of persistence in spatially extended nonequilibrium systems [58]. Exact results were recently obtained on local times for projected observables in stochastic many-body systems $[59,60]$, which provided insight into the emergence of memory on the level of individual non-Markovian trajectories. Notwithstanding, a general approach to fluctuations in time-average statistical mechanics for arbitrary initial conditions remains elusive.

Here, we present a spectral-theoretic approach to finite time-average statistical mechanics of ergodic systems. In mathematical terms, we focus on the statistics of bounded, local, additive functionals of normal ergodic Markovian stochastic processes with continuous and discrete statespaces, including functionals of their (non-Markovian) lowerdimensional projections. The paper is organized as follows. We first provide in Sec. II a brief introduction into timeaverage statistical mechanics. In Sec. III we rederive the well-known Feynman-Kac formulas for Markovian diffusion using Itô calculus. In Sec. IV A spectral theory combined 


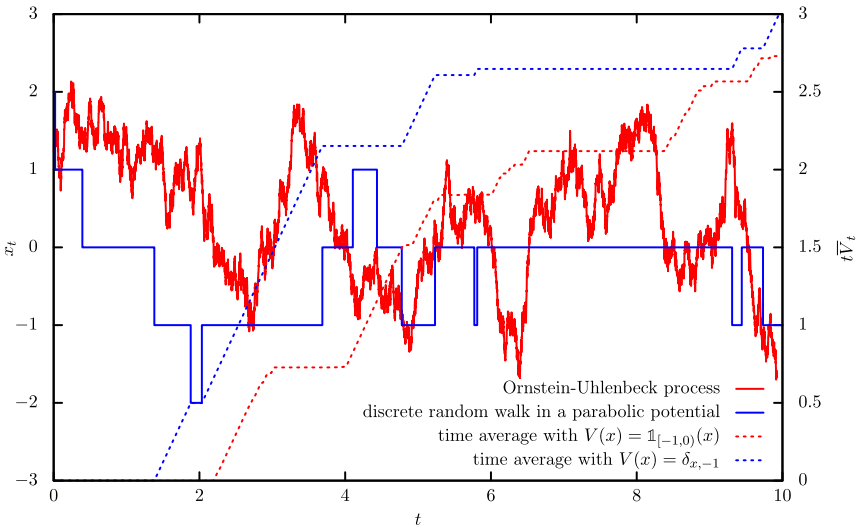

FIG. 1. Realization of a trajectory $\mathbf{x}_{t}$ (solid lines) of a continuous Ornstein-Uhlenbeck diffusion (red) and a Markovian discretespace continuous-time random walk in a quadratic potential (blue). The dotted lines refer to the time average, Eq. (3), with $t \bar{V}_{t}=$ $\int_{0}^{t} V\left(x_{\tau}\right) d \tau$, where we chose $V(x)=\mathbb{1}_{[0,1)}(x)$ for the OrnsteinUhlenbeck and $V(x)=\delta_{-1, x}$ for the discrete random walk.

with non-Hermitian perturbation theory is applied to obtain our main result-exact expressions for the mean, variance, and correlations of time-average observables for any nonstationary preparation of the system, expressed explicitly in terms of the eigenspectrum of the underlying generator of the dynamics, which may correspond to Fokker-Planck diffusion or Markovian dynamics governed by a master equation. We demonstrate explicitly the emergence of a central limit law in a spectral representation on large-deviation timescales. In Sec. IV C we derive our second main result-a general upper bound on fluctuations of occupation measures in terms of an integral of the, generally non-Markovian, return probability that is valid for generators of overdamped dynamics obeying detailed balance. Finally, simple analytically solvable examples are provided in Sec. V to demonstrate how to apply the theory. We conclude in Sec. VI.

\section{TIME-AVERAGE STATISTICAL MECHANICS}

\section{A. Ensemble- versus time-average observables}

Traditional (classical) ensemble statistical mechanics describes physical observations as averages over individual realizations of the dynamics at single (or multiple) predetermined times. For example, the ensemble average of an observable $V\left(\mathbf{x}_{t}\right)$ at a time $t$ for an ergodic stochastic process $\mathbf{x}_{\tau}(0 \leqslant \tau \leqslant t)$ starting from some nonstationary initial condition $p_{0}\left(\mathbf{x}_{0}\right)$ is defined by

$$
\left\langle V\left(\mathbf{x}_{t}\right)\right\rangle_{p_{0}} \equiv \begin{cases}\int_{\Omega} d \mathbf{x} \int_{\Omega} d \mathbf{x}_{0} V(\mathbf{x}) P_{t}\left(\mathbf{x} \mid \mathbf{x}_{0}\right) p_{0}\left(\mathbf{x}_{0}\right), & \Omega \subset \mathbb{R}^{d}, \\ \sum_{\mathbf{x} \in \Omega} \sum_{\mathbf{x}_{0} \in \Omega} V(\mathbf{x}) P_{t}\left(\mathbf{x} \mid \mathbf{x}_{0}\right) p_{0}\left(\mathbf{x}_{0}\right), & \Omega \text { discrete },\end{cases}
$$

where $\Omega$ is the state space of the process and $P_{t}\left(\mathbf{x} \mid \mathbf{x}_{0}\right)$ is the so-called propagator, i.e., $P_{t}\left(\mathbf{x} \mid \mathbf{x}_{0}\right) d \mathbf{x}$ (upper line) is the probability that the process is found in $\mathbf{x} \in \Omega$ within the increment $d \mathbf{x}$ at time $t$ given that it started at $t=0$ at $\mathbf{x}_{0}$. Note that if $\mathbf{x}$ is continuously valued ( $\mathbf{x} \in \Omega \subset \mathbb{R}^{d}$; see Fig. 1, solid red line), then $P_{t}\left(\mathbf{x} \mid \mathbf{x}_{0}\right)$ is a probability density, whereas if $\mathbf{x}$ is from a discrete state space $\Omega$ (Fig. 1, solid blue line), the integral in Eq. (1) (upper line) becomes a sum, $\int_{\Omega} d \mathbf{x} \rightarrow \sum_{\mathbf{x} \in \Omega}$, and $P_{t}\left(\mathbf{x} \mid \mathbf{x}_{0}\right)$ becomes a plain probability as shown in the lower line of Eq. (1). If $\mathbf{x}_{0}$ is sampled and averaged over a stationary (invariant) measure, $p\left(\mathbf{x}_{0}\right)=P_{\text {inv }}\left(\mathbf{x}_{0}\right)$, or if $t$ becomes sufficiently (i.e., ergodically) large, $P_{\infty}\left(\mathbf{x} \mid \mathbf{x}_{0}\right) \equiv P_{\text {inv }}(\mathbf{x})$, the ensemble average becomes time-independent,

$$
\langle V\rangle_{\mathrm{inv}} \equiv \int d \mathbf{x} V(\mathbf{x}) P_{\mathrm{inv}}(\mathbf{x}) .
$$

Conversely, in single-molecule dynamics, single-particle tracking, and other related experiments, one probes individual realizations of $\mathbf{x}_{\tau}$ within the interval $0 \leqslant \tau \leqslant t$ and instead analyzes the observation by taking a time-average. Such time-average observables are in general random, fluctuating quantities with nontrivial statistics. For example, for a physical observable $V\left(\mathbf{x}_{t}\right)$, which may correspond to the squared displacement $[61,62]$ or local time $[51,59,60,63]$ in single-particle tracking or the FRET efficiency [5-8] or distance between two optical traps [9-13] in single-molecule fluorescence and force spectroscopy, respectively, the (local) time-average is defined as

$$
\bar{V}_{t} \equiv t^{-1} \int_{0}^{t} V\left(\mathbf{x}_{\tau}\right) d \tau,
$$

and it depends on the entire history of $\mathbf{x}_{\tau}$ until time $t$ (see also the dotted lines in Fig. 1). The statistical evolution of $\bar{V}_{t}$ is therefore a non-Markovian process characterized by the probability density that the random observable $\bar{V}_{t}$ attains, in a given realization of $\mathbf{x}_{\tau}$, the value $v[46,52,53,59,60]$, which is defined as

$$
\mathcal{P}_{t}^{\bar{V}}\left(v \mid \mathbf{x}_{0}\right) \equiv\left\langle\delta\left(v-\bar{V}_{t}\right)\right\rangle_{\mathbf{x}_{0}},
$$

where $\delta(z)$ is the Dirac delta function and $\langle\cdot\rangle_{\mathbf{x}_{0}}$ denotes the average over all paths starting at $\mathbf{x}_{0}$, i.e., $p_{0}(\mathbf{x})=\delta\left(\mathbf{x}-\mathbf{x}_{0}\right)$, and propagating until time $t$. The corresponding result for arbitrary initial conditions $p_{0}\left(\mathbf{x}_{0}\right)$ follows by superposition, i.e., $\mathcal{P}_{t}^{\bar{V}}\left(v \mid p_{0}\right) \equiv \int_{\Omega} \mathcal{P}_{t}^{\bar{V}}\left(v \mid \mathbf{x}_{0}\right) p_{0}\left(\mathbf{x}_{0}\right) d \mathbf{x}_{0}$ (see also Sec. III B).

The random "empirical density" [64] $\theta_{\mathbf{x}}(t)$ determined from a single trajectory in time-average statistical mechanics is the so-called local-time fraction defined as $[21,59,60]$

$$
\theta_{\mathbf{x}}(t) \equiv t^{-1} \int_{0}^{t} d \tau \delta\left(\mathbf{x}-\mathbf{x}_{\tau}\right)
$$

which allows us to rewrite the time average (3) in the form

$$
\bar{V}_{t}=t^{-1} \int_{0}^{t} d \tau \int_{\Omega} d \mathbf{x} \delta\left(\mathbf{x}-\mathbf{x}_{\tau}\right) V(\mathbf{x}) \equiv \int_{\Omega} d \mathbf{x} V(\mathbf{x}) \theta_{\mathbf{x}}(t),
$$

where $\delta\left(\mathbf{x}-\mathbf{x}_{\tau}\right)$ denotes the Dirac delta function if $\mathbf{x} \in \Omega$ is continuous, whereas $\delta\left(\mathbf{x}-\mathbf{x}_{\tau}\right)$ denotes the Kronecker delta if $\mathbf{x} \in \Omega$ is integer-valued. Note that it is often useful to generalize the local-time fraction in a point $\mathbf{x}$ in Eq. (5) to the notion of occupation time within the hypersurface $V(\mathbf{x})=\mathcal{V}$ defined as

$$
\theta_{\mathcal{V}}(t) \equiv t^{-1} \int_{0}^{t} \delta\left(\mathcal{V}-V\left(\mathbf{x}_{\tau}\right)\right) d \tau
$$


Accordingly, we can rewrite Eq. (6) equivalently in terms of $\theta_{\mathcal{V}}(t)$ as

$$
\bar{V}_{t}=t^{-1} \int_{0}^{t} d \tau \int d \mathcal{V} \delta\left(\mathcal{V}-V\left(\mathbf{x}_{\tau}\right)\right) \mathcal{V} \equiv \int d \mathcal{V} \mathcal{V} \theta_{\mathcal{V}}(t)
$$

Because the dynamics $\mathbf{x}_{t}$ is assumed to be ergodic, we have $\lim _{t \rightarrow \infty} \theta_{\mathbf{x}}(t)=P_{\text {inv }}(\mathbf{x})$ and $\lim _{t \rightarrow \infty} \theta_{\mathcal{V}}(t)=P_{\text {inv }}(\mathcal{V})$ $[52,59,60]$, and

$$
\begin{aligned}
& \lim _{t \rightarrow \infty} \bar{V}_{t}=\int d \mathbf{x} V(\mathbf{x}) P_{\text {inv }}(\mathbf{x})=\int d \mathcal{V} \mathcal{V} P_{\text {inv }}(\mathcal{V}) \equiv\langle V\rangle_{\text {inv }}, \\
& \lim _{t \rightarrow \infty} \mathcal{P}_{t}^{\bar{V}}\left(v \mid \mathbf{x}_{0}\right)=\delta\left(v-\langle V\rangle_{\text {inv }}\right),
\end{aligned}
$$

where we have defined the stationary (or invariant) measure of $\bar{V}\left(\mathbf{x}_{t}\right)$, i.e., $P_{\text {inv }}(\mathcal{V}) \equiv \int d \mathbf{x} \delta(\mathcal{V}-V(\mathbf{x})) P_{\text {inv }}(\mathbf{x})$.

Equations (9) reflect the strong law of large numbers on timescales where $\bar{V}_{t}$ for different values of $t$ decorrelate. Moreover, on the so-called large-deviation timescale, i.e., on the timescale that is finite but longer that the longest relaxation time of $\mathbf{x}_{t}$, we find convergence in the mean, $\left\langle\bar{V}_{t_{\mathrm{DD}}}\right\rangle=$ $\langle V\rangle_{\text {inv }}$, and Gaussian fluctuations around the mean value $[45,59,60,65,66]$. For finite, and in particular subergodic (i.e., supralarge-deviation) times, the statistics of $\bar{V}_{t}$ is, however, nontrivial. Below we provide intuition about the local-time fraction from a practical perspective.

\section{B. Local-time fraction as a histogram inferred from a single trajectory}

To gain more intuition about the local-time fraction (or "empirical density"), we consider, as an example, a Brownian particle diffusing in a harmonic potential. A single trajectory starting from $x_{0}=1.2$ is recorded as a function of time (see the full red line in Fig. 1). We are interested in the distribution (i.e., a histogram) of the particle's position $x_{t}$ inferred from a single trajectory of length $t$ (see Fig. 2). Note that in Fig. 2 we consider a histogram with a finite bin-size $\Delta$, which we denote explicitly as $\theta_{x}^{\Delta}(t)$. In this sense, the local-time fraction (5) is simply a mathematical idealization of a histogram, i.e., $\theta_{x}(t)=\theta_{x}^{\Delta \rightarrow 0}(t)$.

If the trajectory is sufficiently long (i.e., $t \rightarrow \infty), \theta_{x}^{\Delta}(t)$ converges, up to small fluctuations of order $1 / \sqrt{t}$, to a Gaussian stationary (invariant) measure $P_{\text {inv }}(x) \propto e^{-x^{2} / 2}$. This convergence is depicted explicitly in Fig. 2(b). According to Eq. (9), this result depends neither on the initial condition $x_{0}$ nor on the particular realization of the trajectory.

We may also infer a histogram of the particle's position from a short trajectory. The resulting histogram $\theta_{x}^{\Delta}(t)$ appears "rough" and far from Gaussian [see the histogram in Fig. 2(a)]. If we were to repeat the analysis for many trajectories and infer an averaged histogram $\left\langle\theta_{x}^{\Delta}(t)\right\rangle$, we would find as well that it deviates strongly from a Gaussian [see the line in Fig. 2(a)] with large fluctuations around the mean, $\delta \theta_{x}^{\Delta}(t) \equiv\left|\theta_{x}^{\Delta}(t)-\left\langle\theta_{x}^{\Delta}(t)\right\rangle\right| \sim\left\langle\theta_{x}^{\Delta}(t)\right\rangle$ [see the histogram in Fig. 2(a)]. Moreover, both the mean histogram $\left\langle\theta_{x}^{\Delta}(t)\right\rangle$ and the fluctuations around the mean, $\delta \theta_{x}^{\Delta}(t)$, depend not only on $t$ but also on the initial position $x_{0}$, where we observe a persistent cusp [Fig. 2(a)].
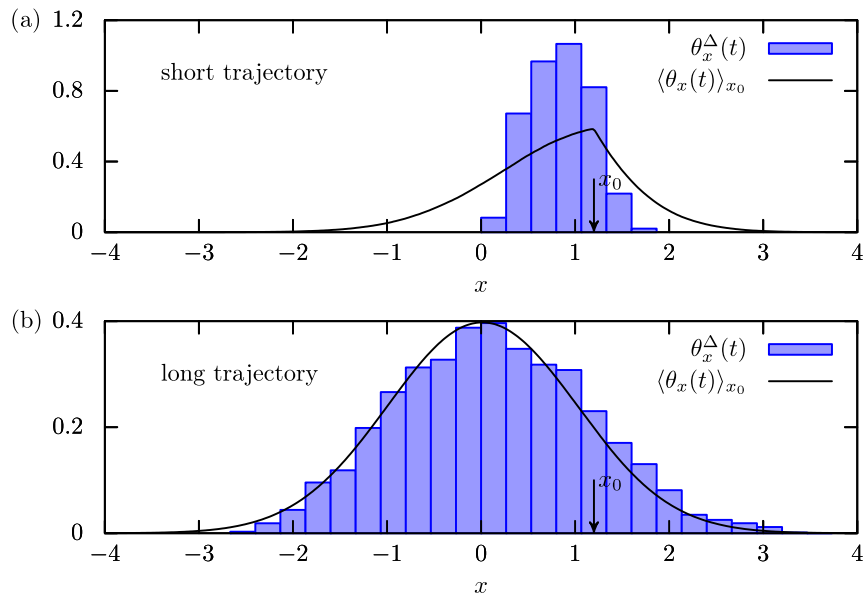

FIG. 2. Local time fraction (or "empirical density") for a Brownian particle in a harmonic potential. The histogram $\theta_{x}^{\Delta}(t)$ (blue) inferred from a single trajectory starting from $x_{0}=1.2$ compared the mean local-time fraction $\left\langle\theta_{y}(t)\right\rangle_{x_{0}}=\lim _{\Delta \rightarrow 0}\left\langle\theta_{y}^{\Delta}(t)\right\rangle_{x_{0}}$. The histogram with bin-size $\Delta$ (here we assume $\Delta=0.3$ ) is defined by $\theta_{x}^{\Delta}(t)=\Delta^{-1} \int_{x-\Delta / 2}^{x+\Delta / 2} \theta_{y}(t) d y$, that is, $\theta_{x}^{\Delta}(t)=\bar{V}_{t}$ with $V(x) \equiv$ $\Delta^{-1} \mathbb{1}_{[x-\Delta / 2, x+\Delta / 2]}(x)$, where "11" denotes the indicator function being 1 if $x \in[x-\Delta / 2, x+\Delta / 2]$ and 0 otherwise. Parameters: $x_{0}=1.2$; (a) $t=1$, (b) $t=100$ (the trajectory is ten times as long as in Fig. 1).

In the reminder of this work, we will focus on the mean values and fluctuations of entries, as well as linear correlations between entries of such (random, realization-dependent) histograms inferred from finite, individual trajectories starting from general initial conditions.

\section{Fluctuations of time averages}

To exploit the role of the local-time fraction $\theta_{\mathbf{x}}(t)$ as a "propagator" in time-average statistical mechanics [via $\bar{V}_{t}=$ $\left.\int_{\Omega} d \mathbf{x} V(\mathbf{x}) \theta_{\mathbf{x}}(t)\right]$, we now relate the statistics of $\theta_{\mathbf{x}}(t)$ to the probability density of a general time-averaged physical observable $\bar{V}_{t}$ defined in Eq. (3). In the example in Fig. 2, $\bar{V}_{t}=\theta_{x}^{\Delta}$ accounts for the value of the histogram at position $x$. The characteristic function of $\bar{V}_{t}$, i.e., the Laplace transform $\tilde{\mathcal{P}}_{t}^{\bar{V}}\left(u \mid \mathbf{x}_{0}\right) \equiv \int_{0}^{\infty} e^{-u v} \mathcal{P}_{t}^{\bar{V}}\left(v \mid \mathbf{x}_{0}\right) d v$ if $v \geqslant 0$, reads

$$
\tilde{\mathcal{P}}_{t}^{\bar{V}}\left(u \mid \mathbf{x}_{0}\right)=\left\langle e^{-u \bar{V}_{t}}\right\rangle_{\mathbf{x}_{0}}=\left\langle e^{-u \int_{\Omega} V(\mathbf{x}) \theta_{\mathbf{x}}(t) d \mathbf{x}}\right\rangle_{\mathbf{x}_{0}},
$$

where we have used Eq. (6) to arrive at the second equality. Equation (10) relates the statistics of the time average $\bar{V}_{t}$ to the statistics of all local-time fractions $\theta_{\mathbf{x}}(t)(\mathbf{x} \in \Omega)$. We note that Eq. (10) must be modified if $V(\mathbf{x})$ can also become negative such that a Fourier transform is required instead, which amounts to replacing $u \rightarrow i \omega$ with $\omega \in \mathbb{R}$ in Eq. (10). The probability density is obtained from Eq. (10) by Laplace inversion,

$$
\mathcal{P}_{t}^{\bar{V}}\left(v \mid \mathbf{x}_{0}\right)=\frac{1}{2 \pi \mathrm{i}} \int_{c-i \infty}^{c+i \infty} e^{u v} \tilde{\mathcal{P}}_{t}^{\bar{V}}\left(u \mid \mathbf{x}_{0}\right) d u,
$$

where $c \in \mathbb{R}$ lies to the right of all singularities of $\tilde{\mathcal{P}}_{t}^{\bar{V}}\left(u \mid \mathbf{x}_{0}\right)$ and we have assumed that $\mathcal{P}_{t}^{\bar{V}}\left(\nu \mid \mathbf{x}_{0}\right)$ is of exponential order for sufficiently large $v$ [in the following section, the conditions on $V\left(\mathbf{x}_{\tau}\right)$ will be made more precise]. If the support extends to 
negative values, Eq. (11) becomes the inverse Fourier transform. Here we are particularly interested in fluctuations of time averages $\bar{V}_{t}$ and $\bar{W}_{t}$ of different physical observables $V\left(\mathbf{x}_{\tau}\right)$ and $W\left(\mathbf{x}_{\tau}\right)$, which are quantified by $[59,60]$

$$
\begin{aligned}
\sigma_{\bar{V}}^{2}(t) & \equiv\left\langle\bar{V}_{t}^{2}\right\rangle_{\mathbf{x}_{0}}-\left\langle\bar{V}_{t}\right\rangle_{\mathbf{x}_{0}}^{2}, \\
C_{\overline{V W}}(t) & \equiv\left\langle\bar{V}_{t} \bar{W}_{t}\right\rangle_{\mathbf{x}_{0}}-\left\langle\bar{V}_{t}\right\rangle_{\mathbf{x}_{0}}\left\langle\bar{W}_{t}\right\rangle_{\mathbf{x}_{0}},
\end{aligned}
$$

where $\sigma_{V}^{2}(t)=C_{\overline{V V}}(t)$ denotes the variance of $\bar{V}_{t}$, and $C_{\overline{V W}}(t)$ denotes the covariance between $\bar{V}_{t}$ and $\bar{W}_{t}$.

In the example in Fig. 2, where $\bar{V}_{t}=\theta_{x}^{\Delta}(t)$ corresponds to the entry $x$ in the histogram, $\sigma_{\bar{V}}^{2}(t) \equiv\left\langle\delta \theta_{x}^{\Delta}(t)^{2}\right\rangle$ refers to the variance of said entries between different realizations of the histogram [i.e., the scatter of $\theta_{x}^{\Delta}(t)$ around $\left.\left\langle\theta_{x}(t)\right\rangle\right]$. Analogously, $C_{\overline{V W}}(t)$ accounts for linear correlations between pairs of entries at $x$ and $y, \theta_{x}^{\Delta}(t)$ and $\theta_{y}^{\Delta}(t)$, in a histogram inferred from a single trajectory of length $t$.

Using Eq. (10), we obtain more generally

$$
\begin{aligned}
\left\langle\bar{V}_{t}^{n} \bar{W}_{t}^{m}\right\rangle_{\mathbf{x}_{0}}= & \prod_{i=1}^{n} \int_{\Omega} d \mathbf{x}_{i} V\left(\mathbf{x}_{i}\right) \prod_{j=1}^{m} \int_{\Omega} d \mathbf{y}_{j} W\left(\mathbf{y}_{j}\right) \\
& \times\left\langle\theta_{\mathbf{x}_{1}}(t) \cdots \theta_{\mathbf{x}_{n}}(t) \theta_{\mathbf{y}_{1}}(t) \cdots \theta_{\mathbf{y}_{m}}(t)\right\rangle_{\mathbf{x}_{0}},
\end{aligned}
$$

where $\mathbf{x}_{i}, \mathbf{y}_{j} \in \Omega$, respectively. Thereby, the ensemble average corresponding to the last term in Eq. (13) is obtained by differentiating the characteristic function with respect to the Laplace (or Fourier) variable.

It therefore follows that the fluctuations and (linear) correlations of general time-average observables are fully specified by multipoint correlation functions of the local-time fraction. These are derived on the basis of the Feynman-Kac formalism, which is presented below for continuous diffusion processes. The extension to discrete state dynamics is discussed afterward.

\section{FLUCTUATIONS OF ADDITIVE FUNCTIONALS}

\section{A. Itô approach to Feynman-Kac theory of additive functionals}

It seems to be customary in the physics literature to start from a path-integral approach to Feynman-Kac theory [46] and then to derive a backward Fokker-Planck equation for the characteristic function [52,53]. Here we provide a simple derivation of the "forward" Feynman-Kac theory based on Itô calculus.

We consider a $d$-dimensional Markovian diffusion $\mathbf{x}_{t} \in$ $\Omega \subset \mathbb{R}^{d}$ process in the presence of a drift $\mathbf{F}(\mathbf{x})$ driven by $d$-dimensional Gaussian white noise governed by the Itô equation

$$
d \mathbf{x}_{t}=\mathbf{F}\left(\mathbf{x}_{t}\right) d t+\sigma d \mathbf{W}_{t},
$$

where $\sigma$ is a $d \times d$ noise matrix such that $\mathbf{D}=\sigma \sigma^{T} / 2$ becomes a symmetric positive-(semi)definite diffusion matrix, and $d \mathbf{W}_{t}$ is an increment of a $d$-dimensional Wiener process, such that $\left\langle W_{t}\right\rangle=\mathbf{0}$ and $\left\langle d W_{t, i} d W_{t^{\prime}, j}\right\rangle=\delta\left(t-t^{\prime}\right) \delta_{i j} d t$. We assume throughout that $\mathbf{F}(\mathbf{x})$ is sufficiently confining to ensure that the process $\mathbf{x}_{t}$ is ergodic with a steady-state probability density $P_{\text {inv }}(\mathbf{x})$. Multiplying the time average in Eq. (3) by the trajectory length $t$, we transform the time average to the additive functional

$$
\psi_{t} \equiv t \bar{V}_{t}=\int_{0}^{t} V\left(\mathbf{x}_{\tau}\right) d \tau .
$$

We now consider the joint process of $\mathbf{x}_{t}$ and $\psi_{t}$. According to Itô's lemma, any twice differentiable function $f(\mathbf{x}, \psi)$ with $\mathbf{x}_{t}$ and $\psi_{t}$ defined by Eqs. (14) and (15), respectively, satisfies

$$
\begin{aligned}
d f\left(\mathbf{x}_{t}, \psi_{t}\right)= & {\left[\mathbf{F}\left(\mathbf{x}_{t}\right) \cdot \nabla_{\mathbf{x}} f\left(\mathbf{x}_{t}, \psi_{t}\right)+\nabla_{\mathbf{x}} \cdot \mathbf{D} \nabla_{\mathbf{x}} f\left(\mathbf{x}_{t}, \psi_{t}\right)\right] d t } \\
& +\nabla_{\mathbf{x}} f\left(\mathbf{x}_{t}, \psi_{t}\right) \cdot \sigma d \mathbf{W}_{t}+V\left(\mathbf{x}_{t}\right) \partial_{\psi} f\left(\mathbf{x}_{t}, \psi_{t}\right) d t
\end{aligned}
$$

where we inserted the diffusion matrix $\mathbf{D}=\sigma \sigma^{T} / 2$, and for the last term we used $d \psi_{t}=V\left(\mathbf{x}_{t}\right) d t$, which follows from Eq. (15).

Using Itô's lemma (16), we derive in the following the time evolution of the joint probability density $Q_{t}\left(\mathbf{x}, \psi \mid \mathbf{x}_{0}\right)$ to find the system in state $\mathbf{x}$ and the functional $\psi_{t}$ in Eq. (15) to attain the value $\psi$ at time $t$ given that the system started from $\mathbf{x}_{0}$. For convenience, we first focus on positive functionals $(\psi \geqslant 0)$. Using a test function that vanishes at the boundary $f(\mathbf{x}, 0)=$ 0 , we obtain after some calculations [67]

$$
\begin{aligned}
\frac{d}{d t}\left\langle f\left(x_{t}, \psi_{t}\right)\right\rangle_{\mathbf{x}_{0}} & =\int_{0}^{\infty} d \psi \int_{\Omega} d \mathbf{x} f(\mathbf{x}, \psi) \partial_{t} Q_{t}\left(\mathbf{x}, \psi \mid \mathbf{x}_{0}\right) \\
& =\int_{0}^{\infty} d \psi \int_{\Omega} d \mathbf{x} Q_{t}\left(\mathbf{x}, \psi \mid \mathbf{x}_{0}\right)\left[\mathbf{F}(\mathbf{x}) \cdot \nabla_{\mathbf{x}} f(\mathbf{x}, \psi)+\nabla_{\mathbf{x}} \cdot \mathbf{D} \nabla_{\mathbf{x}} f(\mathbf{x}, \psi)+V(\mathbf{x}) \partial_{\psi} f(\mathbf{x}, \psi)\right] \\
& =\int_{0}^{\infty} d \psi \int_{\Omega} d \mathbf{x} f(\mathbf{x}, \psi)\left[-\nabla_{\mathbf{x}} \cdot \mathbf{F}(\mathbf{x})+\nabla_{\mathbf{x}} \cdot \mathbf{D} \nabla_{\mathbf{x}}-V(\mathbf{x}) \partial_{\psi}\right] Q_{t}\left(\mathbf{x}, \psi \mid \mathbf{x}_{0}\right)
\end{aligned}
$$

which is obtained as follows. In the first line of Eq. (17), we differentiate both sides of the identity $\int_{0}^{\infty} d \mathbf{x} \int d \psi f(\mathbf{x}, \psi) Q_{t}\left(\mathbf{x}, \psi \mid \mathbf{x}_{0}\right)=\left\langle f\left(\mathbf{x}_{t}, \psi_{t}\right)\right\rangle_{\mathbf{x}_{0}}$ with respect to time $t$. To obtain the second line, we inserted Itô's lemma (16) and finally performed an integration by parts. Since Eq. (17) holds for any function $f$ that vanishes at the boundary $\psi=0$, we obtain

$$
\partial_{t} Q_{t}\left(\mathbf{x}, \psi \mid \mathbf{x}_{0}\right)=\left[\hat{L}-V(\mathbf{x}) \partial_{\psi}-V(\mathbf{x}) \delta(\psi)\right] Q_{t}\left(\mathbf{x}, \psi \mid \mathbf{x}_{0}\right),
$$

where we have defined the forward generator $\hat{L}=\nabla_{\mathbf{x}}$. $\mathbf{D} \nabla_{\mathbf{x}}-\nabla_{\mathbf{x}} \cdot \mathbf{F}(\mathbf{x})$ and further introduced a boundary term 
$V(\mathbf{x}) \delta(\psi) Q_{t}\left(\mathbf{x}, 0 \mid \mathbf{x}_{0}\right)$ that vanishes for $\psi>0$ and is derived in the following two steps. First, Eq. (17) holds for all functions $f(\mathbf{x}, \psi)$ with $f(\mathbf{x}, 0)=0$, which immediately gives Eq. (18) without the last term for $\psi>0$ (see also [67]). Second, the last term in Eq. (18) is required to guarantee the conservation of probability $\int_{0}^{\infty} d \psi \int_{\Omega} d \mathbf{x} \partial_{t} Q_{t}\left(\mathbf{x}, \psi \mid \mathbf{x}_{0}\right)=0$, i.e., to correct for the fact that there is a nonzero probability that the functional has a vanishing value $\psi=0$. Finally, performing a Laplace transform of Eq. (18), $\tilde{Q}_{t}\left(\mathbf{x}, u \mid \mathbf{x}_{0}\right) \equiv$ $\int_{0}^{\infty} e^{-u \psi} Q_{t}\left(\mathbf{x}, \psi \mid \mathbf{x}_{0}\right) d \psi=\left\langle\delta\left(\mathbf{x}-\mathbf{x}_{t}\right) e^{-u \psi_{t}}\right\rangle_{\mathbf{x}_{0}}$, we obtain the forward Feynman-Kac partial differential equation for the characteristic function of the joint density of position and $\psi$,

$$
\partial_{t} \tilde{Q}_{t}\left(\mathbf{x}, u \mid \mathbf{x}_{0}\right)=[\hat{L}-u V(\mathbf{x})] \tilde{Q}_{t}\left(\mathbf{x}, u \mid \mathbf{x}_{0}\right),
$$

where $Q_{t}\left(\mathbf{x}, \psi \mid \mathbf{x}_{0}\right)$ is the central object of the "forward" Feynman-Kac approach $[15,46]$.

We now relax the assumption by allowing for a negative support of $\psi_{t}$, which we denote explicitly by $\psi_{t} \rightarrow$ $\Psi_{t}$. In this case, we need not make any additional assumptions on $f\left(\mathbf{x}_{t}, \Psi_{t}\right)$ for $\Psi_{t}=0$ because naturally $\lim _{|\Psi| \rightarrow \infty} Q_{t}\left(\mathbf{x}, \Psi \mid \mathbf{x}_{0}\right)=0$. The lower boundary of integration over $\Psi$ in Eq. (17) is extended to $-\infty$, and the boundary terms resulting from the partial integration vanish as a result of the boundary conditions. The resulting Eq. (17) implies

$$
\partial_{t} Q_{t}\left(\mathbf{x}, \Psi \mid \mathbf{x}_{0}\right)=\left(\hat{L}-V(\mathbf{x}) \partial_{\Psi}\right) Q_{t}\left(\mathbf{x}, \Psi \mid \mathbf{x}_{0}\right),
$$

which upon taking a Fourier transform $\tilde{Q}_{t}\left(\mathbf{x}, \omega \mid \mathbf{x}_{0}\right) \equiv$ $\int_{-\infty}^{\infty} e^{-i \omega \Psi} Q_{t}\left(\mathbf{x}, \Psi \mid \mathbf{x}_{0}\right) d \Psi$ leads to the forward Feynman-Kac partial differential equation,

$$
\partial_{t} \tilde{Q}_{t}\left(\mathbf{x}, \omega \mid \mathbf{x}_{0}\right)=[\hat{L}-i \omega V(\mathbf{x})] \tilde{Q}_{t}\left(\mathbf{x}, \omega \mid \mathbf{x}_{0}\right) .
$$

Note that the generalization to the case of a joint problem for multiple functionals $\psi_{t}^{i}$ with $i=1, \ldots, p$ is straightforward. Introducing the vectorial notation $\boldsymbol{\psi}_{t} \equiv \int_{0}^{t} \mathbf{V}\left(\mathbf{x}_{\tau}\right) d \tau$ with the corresponding Laplace images $\mathbf{u}$, the resulting equation reads

$$
\partial_{t} \tilde{Q}_{t}\left(\mathbf{x}, \mathbf{u} \mid \mathbf{x}_{0}\right)=[\hat{L}-\mathbf{u} \cdot \mathbf{V}(\mathbf{x})] \tilde{Q}_{t}\left(\mathbf{x}, \mathbf{u} \mid \mathbf{x}_{0}\right),
$$

which allows for the computation of higher-order correlation functions (13). What we actually seek is the marginal probability density of $\boldsymbol{\psi}$ given $\mathbf{x}_{0}$ defined by

$$
\mathcal{P}_{t}^{\psi}\left(\psi \mid \mathbf{x}_{0}\right) \equiv \int_{\Omega} Q_{t}\left(\mathbf{x}, \psi \mid \mathbf{x}_{0}\right) d \mathbf{x},
$$

which is also the statement of the Feynman-Kac theorem [15]. Note that the corresponding characteristic function $\tilde{\mathcal{P}}_{t}^{\psi}\left(\mathbf{u} \mid \mathbf{x}_{0}\right)=\int e^{-\mathbf{u} \cdot \psi} \mathcal{P}_{t}^{\psi}\left(\boldsymbol{\psi} \mid \mathbf{x}_{0}\right) d \boldsymbol{\psi} \equiv\left\langle e^{-\mathbf{u} \cdot \psi_{t}}\right\rangle_{\mathbf{x}_{0}}$ is the solution of the "backward" Feynman-Kac problem [52,53]. Moreover, the marginal probability density of $\mathbf{x}$ given $\mathbf{x}_{0}$ corresponds to the plain propagator $P_{t}\left(\mathbf{x} \mid \mathbf{x}_{0}\right) \equiv \int Q_{t}\left(\mathbf{x}, \boldsymbol{\psi} \mid \mathbf{x}_{0}\right) d \boldsymbol{\psi}$, which solves the (forward) Fokker-Planck equation $\left(\partial_{t}-\right.$ $\hat{L}) P_{t}\left(\mathbf{x} \mid \mathbf{x}_{0}\right)=0$ with initial data $P_{0}\left(\mathbf{x} \mid \mathbf{x}_{0}\right)=\delta\left(\mathbf{x}-\mathbf{x}_{0}\right)$.

Note that the characteristic functions $\tilde{\mathcal{P}}_{t}^{\overline{\mathbf{V}}}$ and $\tilde{\mathcal{P}}_{t}^{\psi}$ are equivalent up to a trivial rescaling of the independent variable, i.e.,

$$
\tilde{\mathcal{P}}_{t}^{\overline{\mathbf{V}}}\left(\mathbf{u} \mid \mathbf{x}_{0}\right)=\left\langle e^{-\mathbf{u} \cdot \overline{\mathbf{V}}_{t}}\right\rangle=\left\langle e^{-\mathbf{u} / t \cdot \psi_{t}}\right\rangle=\tilde{\mathcal{P}}_{t}^{\psi}\left(\mathbf{u} / t \mid \mathbf{x}_{0}\right) .
$$

Therefore, once $\tilde{\mathcal{P}}_{t}^{\psi}$ is determined according to the FeynmanKac program, a simple change of scale of the Laplace image $\mathbf{u} \rightarrow \mathbf{u} / t$ delivers $\tilde{\mathcal{P}}_{t}^{\overline{\mathbf{V}}}$.

\section{B. From the forward to the backward Feynman-Kac equation}

For convenience, we henceforth adopt the bra-ket notation, where the "ket" $|h\rangle$ denotes a vector, the "bra" the integral operator $\langle g| \equiv \int_{\Omega} d \mathbf{x} g^{\dagger}(\mathbf{x})$, and the scalar product is defined as $\langle g \mid h\rangle \equiv \int_{\Omega} d \mathbf{x} g^{\dagger}(\mathbf{x}) h(\mathbf{x})$. Introducing, moreover, the "flat" state $|-\rangle \equiv \int_{\Omega} d \mathbf{x}|\mathbf{x}\rangle$ and $\langle-| \equiv \int_{\Omega} d \mathbf{x}\langle\mathbf{x}|$, Eqs. (21) and (22) for a general initial condition $p_{0}(\mathbf{x})$, i.e., $\left|p_{0}\right\rangle=$ $\int_{\Omega} d \mathbf{x}_{0} p_{0}\left(\mathbf{x}_{0}\right)\left|\mathbf{x}_{0}\right\rangle$ and $\left\langle p_{0}\right|=\int_{\Omega} d \mathbf{x}_{0} p_{0}\left(\mathbf{x}_{0}\right)\left\langle\mathbf{x}_{0}\right|$, have the solution [59]

$$
\tilde{\mathcal{P}}_{t}^{\psi}\left(\mathbf{u} \mid p_{0}\right) \equiv\left\langle-\left|e^{t[\hat{L}-\mathbf{u} \cdot \mathbf{V}(\mathbf{x})]}\right| p_{0}\right\rangle=\left\langle p_{0}\left|e^{t\left[\hat{L}^{\dagger}-\mathbf{u} \cdot \mathbf{V}(\mathbf{x})\right]}\right|-\right\rangle
$$

To arrive at the second equality we have used Green's identity, introduced the adjoint (or backward) Fokker-Planck operator $\hat{L}^{\dagger}=\nabla_{\mathbf{x}} \cdot \mathbf{D} \nabla_{\mathbf{x}}+\mathbf{F}(\mathbf{x}) \cdot \nabla_{\mathbf{x}}$, and used that the Laplace transform of a real function $f(t)$ transforms as $\tilde{f}\left(s^{\dagger}\right)=\tilde{f}^{\dagger}(s)$ under complex conjugation. In the following subsection, we show that for Markov-jump processes (25), the theory can be adopted one-to-one.

\section{Markov-jump dynamics and additive functionals}

Markov-jump processes correspond to a discrete statespace $\Omega$ in which the system jumps with a constant rate $w_{\mathbf{x y}}$ from state $\mathbf{x} \in \Omega$ to another state $\mathbf{y} \in \Omega$, such that the propagator $P_{t}\left(\mathbf{x} \mid \mathbf{x}_{0}\right)$ satisfies the master equation

$$
\partial_{t} P_{t}\left(\mathbf{x} \mid \mathbf{x}_{0}\right)=\sum_{\mathbf{y}}\langle\mathbf{x}|\hat{L}| \mathbf{y}\rangle P_{t}\left(\mathbf{y} \mid \mathbf{x}_{0}\right),
$$

where $\langle\mathbf{x}|\hat{L}| \mathbf{y}\rangle=w_{\mathbf{y x}}$ if $\mathbf{x} \neq \mathbf{y}$ and $\langle\mathbf{x}|\hat{L}| \mathbf{x}\rangle=-\sum_{\mathbf{y} \neq \mathbf{x}}\langle\mathbf{y}|\hat{L}| \mathbf{x}\rangle$ such that $-\langle\mathbf{x}|\hat{L}| \mathbf{x}\rangle>0$ is the rate of leaving state $\mathbf{x}$. According to the celebrated Gillespie algorithm [68], a single trajectory $\mathbf{x}_{\tau}(0 \leqslant \tau \leqslant t)$ consists of a sequence of exponentially distributed local waiting times $\tau_{i}$ in state $\mathbf{x}_{i} \in \Omega$ followed by an instantaneous transition to another state $\mathbf{x}_{i+1} \neq$ $\mathbf{x}_{i}$, with the total time being the sum of waiting times $\sum_{i} \tau_{i}=$ $t-\tau_{R}$, where $\tau_{R}$ is the duration of the final epoch that contains no jump. More precisely, whenever the system is in a state $\mathbf{x}_{i}$ at time $t_{i}$, the probability density to leave said state $\mathbf{x}_{i}$ exactly at time $t_{i+1}=t_{i}+\tau_{i}$ is exponentially distributed with a waiting time density $-\left\langle\mathbf{x}_{i}|\hat{L}| \mathbf{x}_{i}\right\rangle e^{\left\langle\mathbf{x}_{i}|\hat{L}| \mathbf{x}_{i}\right\rangle \tau_{i}}$. After the waiting time, a new (accessible) state, $\mathbf{x}_{i+1}$, is randomly chosen with probability $-\left\langle\mathbf{x}_{i+1}|\hat{L}| \mathbf{x}_{i}\right\rangle /\left\langle\mathbf{x}_{i}|\hat{L}| \mathbf{x}_{i}\right\rangle$. Therefore, the joint probability density that the system, starting from state $\mathbf{x}_{i}$, jumps after time $\tau_{i}$ and the following state is $\mathbf{x}_{i+1}$ becomes $\left\langle\mathbf{x}_{i+1}|\hat{L}| \mathbf{x}_{i}\right\rangle e^{\left\langle\mathbf{x}_{i}|\hat{L}| \mathbf{x}_{i}\right\rangle \tau_{i}}$.

Denoting the number of transitions from state $\mathbf{x}$ to state $\mathbf{y}$ until a time $t$ by $n_{\mathbf{x y}}(t)=\sum_{i} \delta_{\mathbf{x}_{i}, \mathbf{x}} \delta_{\mathbf{x}_{i+1}, \mathbf{y}}$ and identifying the sum of all local waiting times in state $\mathbf{x}$ up to time $t$ by $t \theta_{\mathbf{x}}(t)=\sum_{i} \delta_{\mathbf{x}_{i}, \mathbf{x}} \tau_{i}$, the path probability (or path weight) of $\mathbf{x}_{\tau}(0 \leqslant \tau \leqslant t)$ starting from $\mathbf{x}_{0}$ generated by the Markov dynamics (26) can be written as [37]

$$
P\left(\left\{\mathbf{x}_{\tau}\right\} \mid \mathbf{x}_{0}\right)=\prod_{\mathbf{x} \neq \mathbf{y}}[\langle\mathbf{x}|\hat{L}| \mathbf{y}\rangle]^{n_{\mathbf{y} \mathbf{x}}(t)} e^{\sum_{\mathbf{x}} t \theta_{\mathbf{x}}(t)\langle\mathbf{x}|\hat{L}| \mathbf{x}\rangle} .
$$

Replacing the integral in Eq. (6) by a sum, $\bar{V}_{t}=$ $\sum_{\mathbf{x}} V(\mathbf{x}) \theta_{\mathbf{x}}(t)$, allows us to identify the characteristic function 
of $\psi_{t}=t \bar{V}_{t}$ by [37]

$$
\begin{aligned}
\tilde{\mathcal{P}}_{t}^{\psi}\left(u \mid \mathbf{x}_{0}\right) & \equiv\left\langle e^{-u t \bar{V}_{t}}\right\rangle_{\mathbf{x}_{0}} \\
& =\int d\left\{\mathbf{x}_{\tau}\right\} P\left(\left\{\mathbf{x}_{\tau}\right\} \mid \mathbf{x}_{0}\right) e^{-u \sum_{\mathbf{x}} V(\mathbf{x}) t \theta_{\mathbf{x}}(t)} \\
& =\left\langle-\left|e^{\hat{L}(u) t}\right| \mathbf{x}_{0}\right\rangle,
\end{aligned}
$$

where in the second line we inserted the path weight Eq. (27). While passing from the second to the third line, we tilted the diagonal of the generator in the path weight (27) according to $\langle\mathbf{x}|\hat{L}(u)| \mathbf{x}\rangle \equiv\langle\mathbf{x}|\hat{L}| \mathbf{x}\rangle-u V(\mathbf{x})$, which effectively moves $e^{-u t \bar{V}_{t}}$ into the tilted path weight. In other words, identifying $\hat{L}(u)$ in the second line of Eq. (28) yields the third line. Note that the off-diagonal elements of the tilted generator remain unchanged, that is, $\langle\mathbf{x}|\hat{L}(u)| \mathbf{y}\rangle \equiv\langle\mathbf{x}|\hat{L}| \mathbf{y}\rangle$ if $\mathbf{x} \neq \mathbf{y}$. Since all elements of $\hat{L}$ and $V(\mathbf{x})$ are real, Eq. (25) holds also for Markov-jump processes. As shown in Eq. (24), the characteristic function of $\bar{V}_{t}=\psi_{t} / t$ follows from a trivial change of scale, $\tilde{\mathcal{P}}_{t}^{\bar{V}}\left(u \mid \mathbf{x}_{0}\right)=\tilde{\mathcal{P}}_{t}^{\psi}\left(u / t \mid \mathbf{x}_{0}\right)$. In the following, we develop a spectral theory, which unifies diffusion processes and Markov-jump processes.

\section{Spectral theory of non-Hermitian generators}

We henceforth employ a spectral-theoretic approach and are thus required to make some more specific assumptions about the underlying dynamics in order to assure that the generator $\hat{L}$ is diagonalizable. An excellent account of the theory for Markov-jump dynamics governed by a discrete-state master equation can be found in [69]. In the case of FokkerPlanck dynamics, we consider that $\mathbf{x}_{t}$ is an ergodic Markovian diffusion evolving according to Eq. (14) with the drift field $\mathbf{F}(\mathbf{x})$ not necessarily corresponding to a potential field (which thus includes systems with a broken detailed balance) but at the same time requiring that it is sufficiently confining, that is, it grows sufficiently fast as $|\mathbf{x}| \rightarrow \infty$ to assure that $\hat{L}$ has a pure point-spectrum. Moreover, we require that $\hat{L}$ is diagonalizable, and it can be shown that any normal operator $\hat{L}$, satisfying $\hat{L} \hat{L}^{\dagger}=\hat{L}^{\dagger} \hat{L}$, is in fact diagonalizable [70]. A more detailed mathematical exposé of the requirements for, and properties of, $\hat{L}$ can be found in [60]. In all practical examples presented below, we will in fact assume that the dynamics is overdamped. Moreover, except for the example presented in Sec. VC where detailed balance is violated, $\hat{L}$ will be assumed to obey detailed balance [67,71], implying that it is orthogonally equivalent to a self-adjoint operator and hence automatically diagonalizable.

Let $-\lambda_{k}\left[\operatorname{Re}\left(\lambda_{k}\right) \geqslant 0\right],\left\langle L_{k}\right|$, and $\left|R_{k}\right\rangle$ denote the eigenvalue and orthonormal left and right eigenstates of $\hat{L}$, and let $-\lambda_{k}^{\dagger},\left\langle R_{k}\right|$, and $\left|L_{k}\right\rangle$ denote the corresponding orthonormal eigenstates of $\hat{L}^{\dagger}$ [60], i.e., $\left\langle L_{k} \mid R_{l}\right\rangle=\left\langle R_{k} \mid L_{l}\right\rangle=\delta_{k l}$, with the resolution of identity $\sum_{k}\left|R_{k}\right\rangle\left\langle L_{k}\left|\equiv \sum_{k}\right| L_{k}\right\rangle\left\langle R_{k}\right|=\mathbf{1}$. Then written in the respective eigenbases, $\hat{L}$ and $\hat{L}^{\dagger}$ read

$$
\hat{L}=-\sum_{k} \lambda_{k}\left|R_{k}\right\rangle\left\langle L_{k}\left|, \quad \hat{L}^{\dagger}=-\sum_{k} \lambda_{k}^{\dagger}\right| L_{k}\right\rangle\left\langle R_{k}\right|,
$$

with the ground-state eigenvalue $\lambda_{0}=0$ and the corresponding null-space $\left|R_{0}\right\rangle \equiv\left|P_{\text {inv }}\right\rangle$ and $\left\langle L_{0}\right| \equiv\langle-|$. In the respective dual eigenbasis the propagator $P_{t}\left(\mathbf{x} \mid \mathbf{x}_{0}\right) \equiv\left\langle\mathbf{x}\left|e^{\hat{L} t}\right| \mathbf{x}_{0}\right\rangle=$
$\left\langle\mathbf{x}_{0}\left|e^{\hat{L}^{\dagger} t}\right| \mathbf{x}\right\rangle$ reads

$$
\begin{aligned}
P_{t}\left(\mathbf{x} \mid \mathbf{x}_{0}\right) & =\sum_{k}\left\langle\mathbf{x} \mid R_{k}\right\rangle\left\langle L_{k} \mid \mathbf{x}_{0}\right\rangle e^{-\lambda_{k} t} \\
& \equiv \sum_{k}\left\langle\mathbf{x}_{0} \mid L_{k}\right\rangle\left\langle R_{k} \mid \mathbf{x}\right\rangle e^{-\lambda_{k}^{\dagger} t},
\end{aligned}
$$

where $\left\langle\mathbf{x} \mid R_{k}\right\rangle \equiv R_{k}(\mathbf{x})$ and $\left\langle L_{k}\right| \mathbf{x}_{0} \equiv L_{k}^{\dagger}\left(\mathbf{x}_{0}\right)$, while $\left\langle\mathbf{x}_{0} \mid L_{k}\right\rangle=$ $L_{k}\left(\mathbf{x}_{0}\right)$ and $\left\langle R_{k} \mid \mathbf{x}\right\rangle=R_{k}^{\dagger}(\mathbf{x})$. For overdamped systems with an invertible diffusion matrix $\mathbf{D}$ that obey detailed balance, i.e., $\mathbf{D}^{-1} \mathbf{F}(\mathbf{x})=-\beta \nabla_{\mathbf{x}} U(\mathbf{x})$ with inverse thermal energy $\beta=1 / k_{\mathrm{B}} T$, all $\lambda_{k}$ are real, $\left|P_{\text {inv }}\right\rangle=\left|P_{\text {eq }}\right\rangle$ is the BoltzmannGibbs equilibrium $P_{\mathrm{eq}}(\mathbf{x})=e^{-\beta U(\mathbf{x})} / \int_{\Omega} e^{-\beta U(\mathbf{x})} d \mathbf{x}$, and $\left|L_{k}\right\rangle=e^{\beta U(\mathbf{x})}\left|R_{k}\right\rangle[67,72]$.

\section{CHARACTERISTIC FUNCTION NEAR ZERO VIA NON-HERMITIAN PERTURBATION THEORY}

Based on Eqs. (10) and (13), we only require the momentgenerating function (25) in the limit $|\mathbf{u}| \rightarrow \mathbf{0}$ to calculate moments of arbitrary order. Moreover, recall that $\tilde{\mathcal{P}}_{t}^{\overline{\mathbf{V}}}(\mathbf{u})=$ $\tilde{\mathcal{P}}_{t}^{\psi}(\mathbf{u} / t)$ [see Eq. (24)]. To keep the treatment general, we utilize the spectral expansion of $\hat{L}\left(\hat{L}^{\dagger}\right.$, respectively). We employ perturbation theory to derive the moment-generating function (25) in the limit $|\mathbf{u}| \rightarrow \mathbf{0}$. There are (at least) two possible ways to arrive at the result: a Dyson series approach, which is presented in Appendix B, and by means of secondorder non-Hermitian perturbation theory, which is detailed below. While both yield equivalent results, the perturbationtheoretic approach is more general as it provides a (bi)spectral expansion of the perturbed generator $\hat{L}-\mathbf{u} \cdot \mathbf{V}(\mathbf{x})\left[\hat{L}^{\dagger}-\mathbf{u}\right.$. $\mathbf{V}(\mathbf{x})$, respectively] to second order in $|\mathbf{u}|$. These perturbationtheoretic results, which in the physics literature appear to be new, are applicable beyond time-average statistical mechanics in diverse problems involving perturbations of non-Hermitian and/or non-self-adjoint eigenvalue problems.

Our aim is to diagonalize the "tilted" propagator in Eq. (25) in the limit when $\mathbf{u}$ vanishes. Because $\hat{L}$ is in general not self-adjoint, we need to separately perturb left and right eigenstates. First we must confirm that the tilted propagator $\hat{L}(\mathbf{u}) \equiv$ $\hat{L}-\mathbf{u} \cdot \mathbf{V}(\mathbf{x})$ [and $\hat{L}^{\dagger}(\mathbf{u}) \equiv \hat{L}^{\dagger}-\mathbf{u} \cdot \mathbf{V}(\mathbf{x})$, respectively] is actually diagonalizable in an arbitrarily small neighborhood of $\mathbf{u}=\mathbf{0}$. We focus first on the case in which $V(\mathbf{x}) \geqslant 0$. We Laplace-transform Eq. (25), $t \rightarrow s$, yielding

$$
\tilde{\mathcal{P}}_{s}^{\psi}\left(\mathbf{u} \mid p_{0}\right) \equiv\left\langle-\left|[s-\hat{L}(\mathbf{u})]^{-1}\right| p_{0}\right\rangle=\left\langle p_{0}\left|\left[s-\hat{L}^{\dagger}(\mathbf{u})\right]^{-1}\right|-\right\rangle .
$$

The singularities of Eq. (31) correspond to the perturbed eigenvalue spectrum $\left\{-\lambda_{k}(\mathbf{u})\right\}$ of $\hat{L}(\mathbf{u})$, and diagonalizability is broken whenever one or more singularities are not simple poles (see, e.g., [73]). Equation (25) shows that $|\mathbf{u}|=\mathbf{0}$ is not an accumulation point. Moreover, $\hat{L}$ has a pure point spectrum, therefore an arbitrarily small $|\mathbf{u}|$ cannot cause the emergence of poles of second order in Eq. (31) that would break diagonalizability, akin to the "avoided crossing theorem." Therefore, in the limit $|\mathbf{u}| \rightarrow \mathbf{0}$ the tilted generator $\hat{L}(\mathbf{u})$ is diagonalizable, $\mathbf{u}$ can be taken as real [74], and the eigenspectrum of $\hat{L}(\mathbf{u})$ corresponds to a regular perturbation of the original eigenvalue problem $\hat{L}\left|R_{k}\right\rangle=-\lambda_{k}\left|R_{k}\right\rangle\left(\hat{L}^{\dagger}\left|L_{k}\right\rangle=-\lambda_{k}^{\dagger}\left|L_{k}\right\rangle\right)$, and we seek a perturbative expansion of the tilted eigenspectrum, e.g., of 
$\hat{L}^{\dagger}(\mathbf{u})$ :

$$
\begin{aligned}
-\lambda_{k}^{\dagger^{\prime}}(\mathbf{u}) & =-\lambda_{k}^{\dagger}-\sum_{i>0} \mathbf{u}^{i} \cdot \lambda_{k}^{(i)}, \\
\left|L_{k}(\mathbf{u})\right\rangle & =\left|L_{k}\right\rangle+\sum_{i>0} \mathbf{u}^{i} \cdot\left|\mathbf{L}_{\mathbf{k}}^{\mathbf{i}}\right\rangle, \\
\left\langle R_{k}(\mathbf{u})\right| & =\left\langle R_{k}\left|+\sum_{i>0} \mathbf{u}^{i} \cdot\right| \mathbf{R}_{\mathbf{k}}^{\mathbf{i}}\right\rangle .
\end{aligned}
$$

Without loss of generality, we will henceforth assume that $\mathbf{u}$ is real. Note that while the spectra of $\hat{L}$ and $\hat{L}^{\dagger}$ are complex conjugates [see Eq. (29)], the perturbation is in fact symmetric [see Eq. (31)]. Therefore, the spectra of $\hat{L}(u)$ and $\hat{L}^{\dagger}(u)$ are not complex conjugates except for the unperturbed part, which we denoted in Eq. (32) by quotation marks $\lambda^{\prime \iota^{\prime}}$. According to Eq. (32), multiple functionals yield additive perturbations. It thus suffices to carry out the calculations for $\mathbf{u} \rightarrow u$ and write the corresponding general result by inspection. We are interested in up to second-order moments (13), and therefore we need to evaluate the perturbation up to second order in $u$ :

$$
\begin{aligned}
& \left(-\hat{L}^{\dagger}+u V\right) \sum_{n=0}^{2} u^{n}\left|L_{k}^{n}\right\rangle=\sum_{n=0}^{2} u^{n} \lambda_{k}^{(n)} \sum_{m=0}^{2} u^{m}\left|L_{k}^{m}\right\rangle, \\
& \sum_{n=0}^{2} u^{n}\left\langle R_{k}^{n}\left|\left(-\hat{L}^{\dagger}+u V\right)=\sum_{n=0}^{2} u^{n} \lambda_{k}^{(n)} \sum_{m=0}^{2} u^{m}\right| R_{k}^{m}\right|,
\end{aligned}
$$

where we have adopted the convention $\lambda_{k}^{(0)} \equiv \lambda_{k}^{\dagger},\left\langle R_{k}^{0}\right| \equiv\left\langle R_{k}\right|$, and $\left|L_{k}^{0}\right\rangle \equiv\left|L_{k}\right\rangle$. In Eqs. (34) we only need to keep terms up to $u^{2}$ and equate terms of matching order in $u$. First we impose the preliminary normalization $\left\langle R_{k}(u) \mid L_{k}\right\rangle=\left\langle R_{k} \mid L_{k}(u)\right\rangle=1$, i.e.,

$$
\begin{aligned}
& 1=\left\langle R_{k} \mid L_{k}\right\rangle+u\left\langle R_{k}^{1} \mid L_{k}\right\rangle+u^{2}\left\langle R_{k}^{2} \mid L_{k}\right\rangle+O\left(u^{3}\right), \\
& 1=\left\langle R_{k} \mid L_{k}\right\rangle+u\left\langle R_{k} \mid L_{k}^{1}\right\rangle+u^{2}\left\langle R_{k} \mid L_{k}^{2}\right\rangle+O\left(u^{3}\right),
\end{aligned}
$$

which implies

$$
\left\langle R_{k}^{n} \mid L_{k}\right\rangle=\left\langle R_{k} \mid L_{k}^{n}\right\rangle=0 \text { for } n>0 .
$$

The zeroth order of the expansion gives the solution of the unperturbed system. For the higher orders we need to solve Eqs. (34) matching terms of equal order. Introducing the coupling elements $V_{l k} \equiv\left\langle R_{l}|V| L_{k}\right\rangle$, we obtain (details of the calculation are shown in Appendix A)

$$
\begin{aligned}
& \lambda_{k}^{(1)}=V_{k k}, \quad \lambda_{k}^{(2)}=\sum_{l \neq k} \frac{V_{k l} V_{l k}}{\lambda_{k}^{\dagger}-\lambda_{l}^{\dagger}}, \\
& \left|L_{k}^{1}\right\rangle=\sum_{l \neq k} \frac{V_{l k}}{\lambda_{k}^{\dagger}-\lambda_{l}^{\dagger}}\left|L_{l}\right\rangle, \quad\left\langle R_{k}^{1}\right|=\sum_{l \neq k} \frac{V_{k l}}{\lambda_{k}^{\dagger}-\lambda_{l}^{\dagger}}\left\langle R_{l}\right|, \\
& \left|L_{k}^{2}\right\rangle=\sum_{l \neq k}\left[\sum_{i \neq k} \frac{V_{i k} V_{l i}}{\left(\lambda_{k}^{\dagger}-\lambda_{i}^{\dagger}\right)\left(\lambda_{k}^{\dagger}-\lambda_{l}^{\dagger}\right)}-\frac{V_{k k} V_{l k}}{\left(\lambda_{k}^{\dagger}-\lambda_{l}^{\dagger}\right)^{2}}\right]\left|L_{l}\right\rangle, \\
& \left\langle R_{k}^{2}\right|=\sum_{l \neq k}\left[\sum_{i \neq k} \frac{V_{k i} V_{i l}}{\left(\lambda_{k}^{\dagger}-\lambda_{i}^{\dagger}\right)\left(\lambda_{k}^{\dagger}-\lambda_{l}^{\dagger}\right)}-\frac{V_{k k} V_{k l}}{\left(\lambda_{k}^{\dagger}-\lambda_{l}^{\dagger}\right)^{2}}\right]\left\langle R_{l}\right| .
\end{aligned}
$$

However, while they are orthogonal by construction, the resulting perturbed eigenstates are not normalized anymore, i.e., $\left\langle R_{k}(u) \mid L_{k}(u)\right\rangle \neq 1$. Hence, we need to postnormalize them such that

$$
\mathcal{N}_{k}(u)\left\langle R_{k}(u) \mid L_{k}(u)\right\rangle=1,
$$

where from it follows that

$$
\begin{aligned}
\mathcal{N}_{k}(u) & =\left[\left(\left\langle R_{k}\left|+\sum_{i=1}^{2} u^{i}\left\langle R_{k}^{i}\right|\right)\left(\left|L_{k}\right\rangle+\sum_{i=1}^{2} u^{i}\left|L_{k}^{i}\right\rangle\right)\right]^{-1}\right.\right. \\
& =\frac{1}{1+u^{2}\left\langle R_{k}^{1} \mid L_{k}^{1}\right\rangle+O\left(u^{3}\right)} \\
& =1-u^{2} \sum_{i \neq k} \frac{V_{k i} V_{i k}}{\left(\lambda_{k}^{\dagger}-\lambda_{i}^{\dagger}\right)^{2}}+O\left(u^{3}\right) \\
& \equiv 1-u^{2} \mathcal{M}_{k}+O\left(u^{3}\right),
\end{aligned}
$$

where in the last line we have defined $\mathcal{M}_{k}$. We now use the second-order perturbed eigenspectrum to diagonalize the moment-generating function in Eq. (25),

$$
\tilde{\mathcal{P}}_{t}^{\psi}\left(u \mid p_{0}\right)=\sum_{k} \mathcal{N}_{k}(u)\left\langle p_{0} \mid L_{k}(u)\right\rangle\left\langle R_{k}(u) \mid-\right\rangle e^{-\lambda_{k}^{\hat{H}^{\prime}}(u) t},
$$

where moreover

$$
e^{-\lambda_{k}^{\dagger}(u) t}=e^{-\lambda_{k}^{\dagger} t}\left[1-u \lambda_{k}^{(1)} t+u^{2}\left(\lambda_{k}^{(1) 2} t^{2} / 2-\lambda_{k}^{(2)} t\right)\right]+O\left(u^{3}\right)
$$

and $\quad\left\langle R_{k}(u) \mid-\right\rangle=\left\langle R_{k} \mid-\right\rangle+u\left\langle R_{k}^{1} \mid-\right\rangle+u^{2}\left\langle R_{k}^{2} \mid-\right\rangle+O\left(u^{3}\right)$ with the coefficients given by

$$
\begin{aligned}
\left\langle R_{k} \mid-\right\rangle & =\delta_{k 0}, \quad\left\langle R_{k}^{1} \mid-\right\rangle=\frac{V_{k 0}}{\lambda_{k}^{\dagger}}\left(1-\delta_{k 0}\right), \\
\left\langle R_{k}^{2} \mid-\right\rangle & =-\frac{V_{k k} V_{k 0}}{\lambda_{k}^{\dagger 2}}+\sum_{i \neq k} \frac{V_{k i} V_{i 0}}{\lambda_{k}^{\dagger}\left(\lambda_{k}^{\dagger}-\lambda_{i}^{\dagger}\right)}\left(1-\delta_{k 0}\right)
\end{aligned}
$$

and $\left\langle p_{0} \mid L_{k}(u)\right\rangle=\left\langle p_{0} \mid L_{k}\right\rangle+u\left\langle p_{0} \mid L_{k}^{1}\right\rangle+u^{2}\left\langle p_{0} \mid L_{k}^{2}\right\rangle+O\left(u^{3}\right)$, where $\left|L_{k}^{1}\right\rangle$ and $\left|L_{k}^{2}\right\rangle$ are given by Eq. (37). Using Eqs. (37), (39), as well as (41) and (42), the tilted propagator in Eq. (40) to second order in $u$ reads

$$
\tilde{\mathcal{P}}_{t}^{\psi}\left(u \mid p_{0}\right)=1+\sum_{k>0}\left(u C_{k}^{(1)}+u^{2} C_{k}^{(2)}\right) e^{-\lambda_{k}^{\dagger} t}+O\left(u^{3}\right),
$$

where we have introduced the coefficients

$$
\begin{aligned}
C_{k}^{(1)}= & \delta_{k 0}\left(-V_{00} t+\left\langle p_{0} \mid L_{0}^{1}\right\rangle\right)+\left\langle p_{0} \mid L_{k}\right\rangle\left\langle R_{k}^{1} \mid-\right\rangle \\
C_{k}^{(2)}= & \delta_{k 0}\left[\left\langle p_{0} \mid L_{0}\right\rangle\left(\mathcal{M}_{0}+V_{00}^{2} t^{2} / 2-\lambda_{0}^{(2)} t\right)+\left\langle p_{0} \mid L_{0}^{2}\right\rangle\right] \\
& -t\left(\left\langlep_{0}\left|L_{0}^{1}\right| V_{00} \delta_{k 0}-\left\langle p_{0} \mid L_{k}\right\rangle\left\langle R_{k}^{1}|-| V_{k k}\right)\right.\right. \\
& +\left\langle p_{0} \mid L_{k}^{1}\right\rangle\left\langle R_{k}^{1} \mid-\right\rangle+\left\langle p_{0} \mid L_{k}\right\rangle\left\langle R_{k}^{2} \mid-\right\rangle
\end{aligned}
$$

with $\mathcal{M}_{0}$ defined in Eq. (39). In the special case when initial conditions $\mathbf{x}_{0}$ are drawn from the steady-state probability density, i.e., $p_{0}(\mathbf{x})=P_{\text {inv }}(\mathbf{x})$, these equations simplify to

$$
\begin{aligned}
& \left\langle P_{\text {inv }} \mid L_{k}^{2}\right\rangle=\delta_{k 0}, \quad\left\langle P_{\text {inv }} \mid L_{k}^{1}\right\rangle=\frac{V_{0 k}}{\lambda_{k}^{\dagger}}\left(1-\delta_{k 0}\right), \\
& \left\langle P_{\text {inv }} \mid L_{k}^{2}\right\rangle=-\frac{V_{k k} V_{0 k}}{\lambda_{k}^{\dagger 2}}+\sum_{i \neq k} \frac{V_{i k} V_{0 i}}{\lambda_{k}^{\dagger}\left(\lambda_{k}^{\dagger}-\lambda_{i}^{\dagger}\right)}\left(1-\delta_{k 0}\right) .
\end{aligned}
$$

Turning now to the case in which $V(\mathbf{x})$ extends negative values, we must simply replace $u$ by $i \omega$. To obtain moments up to 
second order [i.e., Eq. (12)], we are now left with evaluating derivatives of Eq. (43) with respect to $u$ at $u=0$.

\section{A. Mean value, fluctuations, and correlation functions for general initial conditions}

We can now derive the mean, variance, and covariance of time-average observables. We first focus on the case of a single time-average observable $\bar{V}_{t}=\psi_{t} / t$ [see Eqs. (3) and (15)]. According to Eq. (24), we must make the replacement $u \rightarrow u / t$ in Eq. (43). We will only present the result in terms of the spectrum of the backward generator $\hat{L}^{\dagger}$ since the results corresponding to $\hat{L}$ follow trivially from Eq. (25). Note that $\left\langle p_{0} \mid-\right\rangle=1$ for any normalized initial condition.

To order $u^{0}$, Eq. (43) simply reflects the normalization of $\mathcal{P}_{t}^{\bar{V}}\left(v \mid p_{0}\right)$, i.e., $\tilde{\mathcal{P}}_{t}^{\bar{V}}\left(0 \mid p_{0}\right)=\int_{0}^{\infty} \mathcal{P}_{t}^{\bar{V}}\left(v \mid p_{0}\right) d v=1$ (and equivalently in the case in which the support of $\bar{V}_{t}$ extends to the entire real axis). To order $u^{1}$, Eq. (43) encodes the mean value $\left\langle\bar{V}_{t}\right\rangle_{p_{0}}$ since it follows from Eq. (10) that $-\left.\partial_{u} \tilde{\mathcal{P}}_{t}^{\bar{V}}\left(u \mid p_{0}\right)\right|_{u=0}=$ $\int_{0}^{\infty} \nu \mathcal{P}_{t}^{\bar{V}}\left(\nu \mid p_{0}\right) d \nu \equiv\left\langle\bar{V}_{t}\right\rangle_{p_{0}}$. Thus the mean value of a timeaverage observable $\left\langle\bar{V}_{t}\right\rangle_{p_{0}}$ evolving from an arbitrary initial condition $p_{0}(\mathbf{x})$ is given by

$$
\left\langle\bar{V}_{t}\right\rangle_{p_{0}}=V_{00}+\frac{1}{t} \sum_{k>0} \frac{V_{k 0}}{\lambda_{k}^{\dagger}}\left\langle p_{0} \mid L_{k}\right\rangle\left(1-e^{-\lambda_{k}^{\dagger} t}\right),
$$

with the anticipated ergodic limit $V_{00}=\left\langle\bar{V}_{t \rightarrow \infty}\right\rangle_{p_{0}}=$ $\left\langle P_{\text {inv }}|V|-\right\rangle \equiv \int_{\Omega} V(\mathbf{x}) P_{\text {inv }}(\mathbf{x}) d \mathbf{x}$. The result (46) is equally valid in cases in which $V(\mathbf{x})$ can become negative (as long as it is bounded). Moreover, from Eq. (46) it is easy to discern the large-deviation asymptotic

$$
\lim _{t \gg 1 / \operatorname{Re} \lambda_{1}^{\dagger}}\left\langle\bar{V}_{t}\right\rangle_{p_{0}} \simeq V_{00}+\frac{1}{t} \sum_{k>0} \frac{V_{k 0}}{\lambda_{k}^{\dagger}}\left\langle p_{0} \mid L_{k}\right\rangle,
$$

and in the special case of steady-state initial conditions, $p_{0}\left(\mathbf{x}_{0}\right)=P_{\text {inv }}\left(\mathbf{x}_{0}\right)$, Eq. (46) reduces to the time-independent ergodic result $\left\langle\bar{V}_{t}\right\rangle_{\text {inv }}=V_{00}$. To order $u^{2}$, Eq. (43) encodes the second moment via $\left.\partial_{u}^{2} \tilde{\mathcal{P}}_{t}^{\bar{V}}\left(u \mid p_{0}\right)\right|_{u=0}=\int_{0}^{\infty} v^{2} \mathcal{P}_{t}^{\bar{V}}\left(v \mid \mathbf{x}_{0}\right) d v \equiv$ $\left\langle\bar{V}_{t}^{2}\right\rangle_{p_{0}}$, which reads

$$
\begin{aligned}
\left\langle\bar{V}_{t}^{2}\right\rangle_{p_{0}}= & V_{00}^{2}+\frac{2}{t} \sum_{k>0} \frac{V_{k 0}}{\lambda_{k}^{\dagger}}\left[V_{0 k}+\left\langle p_{0} \mid L_{k}\right\rangle\left(V_{00}+V_{k k} e^{-\lambda_{k}^{\dagger} t}\right)\right] \\
& +\frac{2}{t^{2}} \sum_{k>0} V_{k 0}\left\{\left[\left\langle p_{0} \mid L_{k}\right\rangle\left(V_{k k}-V_{00}\right)-V_{k 0}\right] \frac{\left(1-e^{-\lambda_{k}^{\dagger} t}\right)}{\lambda_{k}^{\dagger 2}}\right. \\
& \left.+\sum_{l>0, l \neq k} \frac{V_{l k}\left\langle p_{0} \mid L_{l}\right\rangle}{\lambda_{k}^{\dagger}-\lambda_{l}^{\dagger}}\left(\frac{1-e^{-\lambda_{l}^{\dagger} t}}{\lambda_{l}^{\dagger}}-\frac{1-e^{-\lambda_{k}^{\dagger} t}}{\lambda_{k}^{\dagger}}\right)\right\},
\end{aligned}
$$

which together with Eq. (46) yields the variance

$$
\sigma_{\bar{V}, p_{0}}^{2}(t) \equiv\left\langle\bar{V}_{t}^{2}\right\rangle_{p_{0}}-\left\langle\bar{V}_{t}\right\rangle_{p_{0}}^{2} .
$$

We further introduce the following notational convention for localized initial conditions $p_{0}(\mathbf{x})=\delta\left(\mathbf{x}-\mathbf{x}_{0}\right), \sigma_{\bar{V}, p_{0}}^{2}(t) \rightarrow$ $\sigma_{\bar{V}, \mathbf{x}_{0}}^{2}(t)$. From Eqs. (46), (48), and (49) follows the anticipated ergodic result $\left\langle\bar{V}_{t \rightarrow \infty}^{2}\right\rangle_{p_{0}}=V_{00}^{2}$, proving that in the ergodic limit $\bar{V}_{t}$ becomes deterministic [i.e., the variance vanishes, $\left.\sigma_{\bar{V}, p_{0}}^{2}(t \rightarrow \infty)=\left\langle\bar{V}_{t \rightarrow \infty}^{2}\right\rangle_{p_{0}}-\left\langle\bar{V}_{t \rightarrow \infty}\right\rangle_{p_{0}}^{2}=0\right]$. Conversely the large-deviation asymptotic reads for any initial condition $p_{0}(\mathbf{x})$,

$$
\lim _{\operatorname{Re} \lambda_{1}^{\dagger} t \gg 1}\left\langle\bar{V}_{t}^{2}\right\rangle_{p_{0}}=V_{00}^{2}+\frac{2}{t} \sum_{k>0} \frac{V_{k 0}}{\lambda_{k}^{\dagger}}\left(V_{0 k}+V_{00}\left\langle p_{0} \mid L_{k}\right\rangle\right),
$$

yielding a large-deviation variance

$$
\lim _{\operatorname{Re} \lambda_{1} t \gg 1} \sigma_{\bar{V}}^{2}(t)=\frac{2}{t} \sum_{k>0} \frac{V_{0 k} V_{k 0}}{\lambda_{k}^{\dagger}}+O\left(t^{-2}\right),
$$

which embodies the emergence of the central-limit theorem. One can further show that all higher cumulants decay to zero faster than $1 / t$. Since the only distribution with a finite number of nonzero cumulants is the Gaussian distribution [75], the large-deviation mean value (47) and variance (51) specify the entire asymptotic probability density for time-average observables along trajectories of length $t \gg 1 / \operatorname{Re} \lambda_{1}^{\dagger}$.

In the special case of steady-state initial conditions, $p_{0}(\mathbf{x})=P_{\text {inv }}(\mathbf{x})$, we find the variance satisfies (see also [59])

$$
\sigma_{\bar{V}, \text { inv }}^{2}(t)=\frac{2}{t} \sum_{k>0} \frac{V_{0 k} V_{k 0}}{\lambda_{k}^{\dagger}}\left(1-\frac{1-e^{-\lambda_{k}^{\dagger} t}}{\lambda_{k}^{\dagger} t}\right) .
$$

Note that for overdamped systems in detailed balance, we have $\lambda_{k}^{\dagger} \in \mathbb{R}$ and $\left|L_{k}\right\rangle=e^{\beta U(\mathbf{x})}\left|R_{k}\right\rangle \in \mathbb{R}$. Therefore, $V_{0 k} V_{k 0} \geqslant$ 0, which implies [compare Eqs. (51) and (52)] that the fluctuations for stationary initial conditions are bounded from above by $\sigma_{\bar{V}, \text { inv }}^{2}(t) \leqslant \lim _{\operatorname{Re} \lambda_{1} t \gg 1} \sigma_{\bar{V}}^{2}(t)$.

We now inspect the correlation between two functionals $\bar{V}_{1, t}$ and $\bar{V}_{2, t}$ [see Eq. (12)] defined as $C_{\bar{V}_{1} \bar{V}_{2}}(t)=$ $\left\langle\bar{V}_{1, t} \bar{V}_{2, t}\right\rangle_{p_{0}}-\left\langle\bar{V}_{1, t}\right\rangle_{p_{0}}\left\langle\bar{V}_{2, t}\right\rangle_{p_{0}}$. The mean values were derived in Eq. (46), so we only require the mixed second moment $\left\langle\bar{V}_{1, t} \bar{V}_{2, t}\right\rangle_{p_{0}}$, which is obtained from the joint momentgenerating function [i.e., generalization of Eq. (40) to two variables] as $\left\langle\bar{V}_{1, t} \bar{V}_{2, t}\right\rangle_{p_{0}}=\left.\partial_{u_{1} u_{2}}^{2} \tilde{\mathcal{P}}_{t}^{\overline{\mathbf{V}}}\left(\mathbf{u} \mid p_{0}\right)\right|_{\mathbf{u}=\mathbf{0}}$. A lengthy calculation leads, upon introducing the coupling elements $U_{k l}^{i} \equiv$ $\left\langle R_{k}\left|V_{i}(\mathbf{x})\right| L_{l}\right\rangle$ and the shorthand notation $W_{k l m n}=U_{k l}^{1} U_{m n}^{2}+$ $U_{k l}^{2} U_{m n}^{1}$, to the exact result

$$
\begin{aligned}
& \left\langle\bar{V}_{1, t} \bar{V}_{2, t}\right\rangle_{p_{0}} \\
& =\frac{W_{0000}}{2}+\frac{1}{t} \sum_{k>0} \frac{1}{\lambda_{k}^{\dagger}}\left(W_{k 00 k}+\left\langle p_{0} \mid L_{k}\right\rangle\left[W_{00 k 0}-W_{k 0 k k} e^{-\lambda_{k}^{\dagger} t}\right]\right) \\
& \quad+\frac{1}{t^{2}} \sum_{k>0}\left\{\left[\left\langle p_{0} \mid L_{k}\right\rangle\left(W_{k 0 k k}-W_{00 k 0}\right)-W_{k 00 k}\right] \frac{\left(1-e^{-\lambda_{k}^{\dagger} t}\right)}{\lambda_{k}^{\dagger 2}}\right. \\
& \left.\quad+\sum_{l>0, l \neq k} \frac{W_{k 0 l k}\left\langle p_{0} \mid L_{l}\right\rangle}{\lambda_{k}^{\dagger}-\lambda_{l}^{\dagger}}\left(\frac{1-e^{-\lambda_{l}^{\dagger} t}}{\lambda_{l}^{\dagger}}-\frac{1-e^{-\lambda_{k}^{\dagger} t}}{\lambda_{k}^{\dagger}}\right)\right\},
\end{aligned}
$$

and we note that $\left\langle\bar{V}_{1, t}\right\rangle_{\text {inv }}\left\langle\bar{V}_{2, t}\right\rangle_{\text {inv }}=W_{0000} / 2$, implying that for an ergodic system, any two functionals asymptotically decorrelate, $\lim _{t \rightarrow \infty} C_{\bar{V}_{1} \bar{V}_{2}}(t)=0$. Equations (46), (48), and (53) expressing the mean value and second moments (and together the variance and covariance) of the time average of a general physical observable $V\left(\mathbf{x}_{\tau}\right)$ of type Eq. (3) solely in 
terms of the eigenspectrum of the underlying generator are the main theoretical result of this work.

In the case of stationary initial conditions $p_{0}(\mathbf{x})=P_{\text {inv }}(\mathbf{x})$, we have that $\left\langle P_{\text {inv }} \mid L_{k}\right\rangle=\delta_{k 0}$, and as a result of Eq. (53) the covariance reduces to (note that $W_{k 00 k}=W_{0 k k 0}$ and $W_{0000} / 2=$ $\left.\left\langle\bar{V}_{1, t}\right\rangle_{\text {inv }}\left\langle\bar{V}_{2, t}\right\rangle_{\text {inv }}\right)$

$$
C_{\bar{V}_{1} \bar{V}_{2}}^{\text {inv }}(t)=\frac{1}{t} \sum_{k>0} \frac{W_{0 k k 0}}{\lambda_{k}^{\dagger}}\left(1-\frac{1-e^{-\lambda_{k}^{\dagger} t}}{\lambda_{k}^{\dagger} t}\right) .
$$

Finally, in the large-deviation regime, we recover

$$
\lim _{t \gg 1 / \lambda_{1}} C_{\bar{V}_{1} \bar{V}_{2}}(t)=\frac{1}{t} \sum_{k>0} \frac{W_{0 k k 0}}{\lambda_{k}^{\dagger}}+O\left(t^{-2}\right),
$$

the $1 / t$ scaling reflecting the emergence of the centrallimit theorem. Therefore, it follows that an arbitrary set of $m$ time-average observables $\overline{\mathbf{V}}_{t}=\left\{\bar{V}_{i, t}\right\}$ in the largedeviation limit exhibits Gaussian statistics. If we denote the vector of mean values as $\langle\overline{\mathbf{V}}\rangle_{\text {inv }}$ and introduce the symmetric covariance matrix $\mathbf{C}$ with diagonal elements $\mathbf{C}_{i i}=$ $t \lim _{t \gg 1 / \operatorname{Re} \lambda_{1}} \sigma_{\bar{V}_{i}}^{2}(t)$ [see Eq. (51)] and off-diagonal elements $\mathbf{C}_{i j}=t \lim _{t \gg 1 / \operatorname{Re} \lambda_{1}} C_{\bar{V}_{i} \bar{V}_{j}}(t)$ [see Eq. (55)], then the probability density that $\overline{\mathbf{V}}_{t}$ attains a value $\boldsymbol{v}$ obeys the asymptotic Gaussian limit law $\lim _{t \gg 1 / \operatorname{Re} \lambda_{1}} \mathcal{P}_{t}^{\overline{\mathbf{V}}}\left(\boldsymbol{v} \mid p_{0}\right) \equiv \mathcal{P}_{t}^{\mathrm{LD}}(\boldsymbol{v})$, where

$$
\mathcal{P}_{t}^{\mathrm{LD}}(\boldsymbol{v}) \simeq \frac{e^{-\frac{1}{2}\left(\boldsymbol{v}-\langle\overline{\mathbf{V}})_{\text {inv }}\right)^{T} \mathbf{C}^{-1}\left(\boldsymbol{v}-\langle\overline{\mathbf{V}}\rangle_{\text {inv }}\right) t}}{\sqrt{(2 \pi)^{m} \operatorname{det} \mathbf{C} / t}}
$$

We now introduce the rescaled variables $\hat{\boldsymbol{v}} \equiv \boldsymbol{v} \sqrt{t}$ and scaled mean $\boldsymbol{\mu} \equiv\left\langle\overline{\mathbf{V}}_{t} \sqrt{t}\right\rangle_{\text {inv }}$, which upon renormalization lead to a time-independent density. Moreover, we define

$$
\Xi=\left(\hat{v}_{i}-\mu_{i}\right) / \sqrt{\mathbf{C}_{i i}}, \quad \Xi_{i \mid j}=\left(\hat{v}_{i}-\tilde{\mu}_{i \mid j}\right) / \tilde{\sigma}_{i \mid j}
$$

with the shifted mean and stretched variance

$$
\begin{aligned}
\tilde{\mu}_{i \mid j} & =\mu_{i}+\left(\hat{v}_{j}-\mu_{j}\right) \mathbf{C}_{i j} / \mathbf{C}_{j j}, \\
\tilde{\sigma}_{i \mid j}^{2} & =\left(\mathbf{C}_{i i} \mathbf{C}_{j j}-\mathbf{C}_{i j}^{2}\right) / \mathbf{C}_{j j} .
\end{aligned}
$$

Then the limit law (56) implies that the univariate large deviations $\mathcal{P}_{t}^{\mathrm{LD}}(\hat{v})$ and conditional bivariate large deviations $\mathcal{P}_{t}^{\mathrm{LD}}\left(\hat{v}_{1} \mid \hat{v}_{2}\right) \equiv \mathcal{P}_{t}^{\mathrm{LD}}\left(\hat{v}_{1}, \hat{v}_{2}\right) / \mathcal{P}_{t}^{\mathrm{LD}}\left(\hat{v}_{2}\right)$ collapse, upon rescaling, onto a universal Gaussian master curve

$$
\begin{gathered}
\sqrt{\frac{\mathbf{C}_{i i}}{t^{1 / 2}}} \mathcal{P}_{t}^{\mathrm{LD}}\left(\hat{v}_{i}\right) \rightarrow \mathcal{N}_{\Xi}(0,1), \\
\frac{\tilde{\sigma}_{i \mid j}}{\sqrt{t}} \mathcal{P}_{t}^{\mathrm{LD}}\left(\hat{v}_{i} \mid \hat{v}_{j}\right) \rightarrow \mathcal{N}_{\Xi_{i \mid j}}(0,1),
\end{gathered}
$$

where $\mathcal{N}_{x}(0,1)$ denotes the Gaussian probability density with zero mean and unit variance. The explicit rescaling [i.e., Eq. (59) combined with Eq. (51) and Eq. (55)] leading to the collapse onto a master unit normal density in the largedeviation limit is the main practical consequence of our large-deviation result.

\section{B. Degenerate eigenspectra}

Note that if the spectrum of $\hat{L}$ has degenerate eigenstates (such as, e.g., in single-file diffusion $[59,60]$ ) special care is required for initial conditions that do not correspond to the steady state, i.e., $p_{0}(\mathbf{x}) \neq P_{\text {inv }}(\mathbf{x})$, as a result of the singularities the degeneracy causes in Eqs. (48) and (53). As is customary in regular perturbation theory (see, e.g., [76]), one must first postdiagonalize all the respective degenerate subspaces prior to using Eqs. (48) and (53). Once this has been taken care of (using any of the many possible methods [77]) and the degenerate eigenstates are replaced by their appropriate linear combinations, Eqs. (48)-(53) can be used as they stand.

\section{A general upper bound for occupation measures for overdamped reversible dynamics}

When $\hat{L}$ corresponds to reversible overdamped dynamics [i.e., $\mathbf{D}^{-1} \mathbf{F}(\mathbf{x})=-\beta \nabla_{\mathbf{x}} U(\mathbf{x})$ is a gradient field], or to a reversible Markov-jump process [i.e., the transition matrix elements in Eq. (26) satisfy the symmetry $\langle\mathbf{y}|\hat{L}| \mathbf{x}\rangle /\langle\mathbf{x}|\hat{L}| \mathbf{y}\rangle=$ $\left.e^{\beta U(\mathbf{x})-\beta U(\mathbf{y})}\right]$, the large-deviation asymptotic (51) provides an upper bound for fluctuations of the occupation time fraction in any subdomain $\mathcal{V} \subseteq \Omega, V(\mathbf{x})=\mathcal{V}$, for any duration of the trajectory [which naturally includes the local-time fraction when $V(\mathbf{x})=\mathbf{x}]$.

Let us define the projection operator $\hat{\Gamma}_{\mathbf{x}}(\mathcal{V} ; V) \equiv$ $\int_{\Omega} d \mathbf{x} \delta(\mathcal{V}-V(\mathbf{x}))$ [60], which projects the full dynamics $\mathbf{x} \subset \mathbb{R}^{d}$ onto the hypersurface compatible with a given value of the observable $V(\mathbf{x})=\mathcal{V}$. Then the (generally non-Markovian) joint probability density that the observable $V(\mathbf{x})$ starts from $\mathcal{V}$ and returns to the initial value $\mathcal{V}$ at time $t$ in an ensemble of trajectories $\mathbf{x}_{t}$ starting from the equilibrium probability density $P_{\text {inv }}\left(\mathbf{x}_{0}\right)=P_{\text {eq }}\left(\mathbf{x}_{0}\right)$ is defined as [60]

$$
G_{t}^{\mathrm{eq}}(\mathcal{V}, \mathcal{V}) \equiv \hat{\Gamma}_{\mathbf{x}}(\mathcal{V} ; V) \hat{\Gamma}_{\mathbf{x}_{0}}(\mathcal{V} ; V) P_{t}\left(\mathbf{x} \mid \mathbf{x}_{0}\right) P_{\mathrm{eq}}\left(\mathbf{x}_{0}\right) .
$$

We now recall the definition of the occupation time fraction of $\mathbf{x}_{\tau}$ within the hypersurface $V(\mathbf{x})=\mathcal{V}$ in Eq. (8). Then Eq. (51) and the spectral decomposition of $P_{t}\left(\mathbf{x} \mid \mathbf{x}_{0}\right)$ in Eq. (30) imply the general upper bound on $\theta_{\mathcal{V}}(t)$,

$$
t \sigma_{\theta \mathcal{V}, \text { inv }}^{2}(t) \leqslant 2 \int_{0}^{\infty}\left[G_{t}^{\mathrm{eq}}(\mathcal{V}, \mathcal{V})-G_{\infty}^{\mathrm{eq}}(\mathcal{V}, \mathcal{V})\right] d t
$$

where equality holds in the limit $t \rightarrow \infty$. Note that in the special case when $V(\mathbf{x})=\mathbf{x}$, Eq. (61) bounds the local-time fraction defined in Eq. (5).

To prove the bound (61), let us express Eq. (60) using the spectral expansion of $\hat{L}^{\dagger}$ (or equivalently $\hat{L}$ ). Since we are considering systems in detailed balance, the eigenspectrum is real. Introducing the elements $V_{k l}(\mathcal{V}) \equiv\left\langle R_{l}|\delta(\mathcal{V}-V(\mathbf{x}))| L_{k}\right\rangle$, the spectral representation of Eq. (60) reads (see also [60])

$$
G_{t}^{\mathrm{eq}}(\mathcal{V}, \mathcal{V})=\sum_{k} V_{0 k}(\mathcal{V}) V_{k 0}(\mathcal{V}) e^{-\lambda_{k} t}
$$

such that $\lim _{t \rightarrow \infty} G_{t}^{\mathrm{eq}} \equiv G_{\infty}^{\mathrm{eq}}(\mathcal{V}, \mathcal{V})=V_{00}(\mathcal{V})^{2}$. Therefore,

$\int_{0}^{\infty}\left[G_{t}^{\mathrm{eq}}(\mathcal{V}, \mathcal{V})-V_{00}(\mathcal{V})^{2}\right] d t=\sum_{k>0} V_{0 k}(\mathcal{V}) V_{k 0}(\mathcal{V}) / \lambda_{k}$

Multiplying Eq. (63) by 2 and dividing by $t$ we obtain Eq. (51) for the case when $\bar{V}_{t}=\theta_{\mathcal{V}}(t)$ defined in Eq. (8), which in turn proves asymptotic equality as $t \rightarrow \infty$. Because for systems obeying detailed balance we further have $\left|L_{k}\right\rangle=e^{\beta U(\mathbf{x})}\left|R_{k}\right\rangle$, 
each coefficient is positive, $V_{0 k}(\mathcal{V}) V_{k 0}(\mathcal{V}) \geqslant 0$, because it corresponds to $e^{-\beta U(\mathbf{x})}>0$ multiplied by the square of a real number. Together with Eq. (52) this proves that the inequality holds for any $t$ and completes the proof of the existence and tightness of the bound (61).

Equation (61) enables us to obtain an upper bound on fluctuations of $\theta_{\mathcal{V}}(t)$ - the (generally non-Markovian) occupation measure that the full dynamics $\mathbf{x}_{\tau}$ along a single trajectory is found within the hypersurface $V(\mathbf{x})=\mathcal{V}$-from the integral over the return probability (60). It thereby also bounds the fluctuations of random time-average "empirical densities," that is, local-time fractions [see Eq. (5)], by means of the corresponding deterministic (ensemble) joint return probability density (60). Equation (61) is the main practical result of this work. Interestingly, a similar bound involving the integral of the return probability has been found in Ref. [78] in the study of large-deviation asymptotics of the first passage times.

\section{Physical interpretation of the results}

We now provide some intuition about the developed theory. As time evolves, the value of $\bar{V}_{t}$ for an ergodic process eventually becomes only weakly correlated. The statistics of $\bar{V}_{t}$ passes first through the large-deviation regime (56), where the central-limit theorem kicks in with Gaussian statistics, and finally ends up in Khinchin's law of large numbers, where it becomes deterministic and equal to $\left\langle\bar{V}_{t}\right\rangle_{\text {inv }}$ [79]. For simplicity, we start in the large-deviation regime (51).

By using spectral theory, we map fluctuations of $\bar{V}_{t}$ onto the eigenmodes of $\hat{L}$ (and/or $\hat{L}^{\dagger}$, respectively), with the "similarity" to a given eigenmode reflected by the overlaps $V_{0 k}, V_{k 0}$. Since on these timescales all memory of the initial condition is lost, which is equivalent to imposing stationary initial conditions, only overlaps from and to the ground state are relevant. Moreover, due to the orthogonality of eigenmodes, these projections are statistically independent. Each eigenmode has a finite lifetime or correlation time $1 / \lambda_{k}$. Therefore, in a time $t \gg \lambda_{k}^{-1}$ any $k$ th projection acts as shot-noise, and there will be $t \lambda_{k}$ independent realizations of such a projection reducing the (co)variance by a factor $1 / t \lambda_{k}$ [see Eqs. (51) and (55)]. In the limit $t \rightarrow \infty$, the Gaussian converges to a Dirac delta, i.e., $\lim _{t \rightarrow \infty} \mathcal{P}_{t}^{\mathrm{LD}}(\boldsymbol{v})=\delta\left(\boldsymbol{v}-\langle\overline{\mathbf{V}}\rangle_{\text {inv }}\right)$.

At shorter times, nontrivial corrections to these largedeviation results arise due to strong correlations between the values of $\bar{V}_{t}$ at different times $t$. As a result of these correlations, the "completely decorrelated" large-deviation results in Eqs. (51) and (55) become reduced by a term that seems to reflect the "effective probability of mode $k$ to persist until $t$," $t^{-1} \int_{0}^{t} e^{-\lambda_{k} \tau} d \tau=\left(1-e^{-\lambda_{k} t}\right) / \lambda_{k} t$ [see Eqs. (52) and (54) as well as Eqs. (B3) and (B4)]. In the case of general initial conditions, $p_{0}(\mathbf{x})$ additional terms arise [see Eqs. (50) and (53)] that reflect the memory of the initial condition. These terms, however, are difficult to interpret beyond the point that they reflect projections that couple different excited eigenstates and thus describe fluctuation modes that are more complicated than simple excursions starting and ending in the steady state.

\section{APPLICATIONS OF THE THEORY}

We now apply the theory to a collection of simple illustrative examples. Due to the fundamental role played by the local-time fraction $\theta_{\mathbf{x}}(t)$ and because it determines the dynamics of other time-average observables [see Eq. (6)], we focus on $\theta_{\mathbf{x}}(t)$ alone. The coupling elements are therefore simply given by $V_{l k}=\int_{\Omega} d \mathbf{y} R_{l}(\mathbf{y}) \delta(\mathbf{x}-\mathbf{y}) L_{k}(\mathbf{y}) \equiv R_{l}(\mathbf{x}) L_{k}(\mathbf{x})$. We first present explicit results for local times for continuous space-time Markovian diffusion processes and an irreversible (i.e., driven) three-state unicyclic network. Next, we apply the theory to a simple two-state Markov model of the celebrated Berg-Purcell problem [28-30,36], i.e., the physical limit to the precision of receptor-mediated measurement of the concentration of ligand molecules.

As minimal, exactly solvable models of continuous-space Markovian diffusion, we consider a Wiener process confined to a unit interval with reflective boundaries and the Ornstein-Uhlenbeck process. To demonstrate the theory for Markov-jump dynamics, we consider a random walk in a finite harmonic potential and a simple three-state unicyclic network.

\section{A. Local time fraction of the Wiener process in the unit interval}

The propagator of the Wiener process confined to a unit interval (i.e., $L=1$ ) is the solution of

$$
\left(\partial_{t}-\partial_{x}^{2}\right) P_{t}^{\mathrm{W}}\left(x \mid x_{0}\right)=0,\left.\partial_{x} P_{t}^{\mathrm{W}}\right|_{x=0}=\left.\partial_{x} P_{t}^{\mathrm{W}}\right|_{x=1}=0,
$$

with initial condition $P_{0}^{\mathrm{W}}\left(x \mid x_{0}\right)=\delta\left(x-x_{0}\right)$. The eigenvalues of $\partial_{x}^{2}$ in a unit interval are given by $\lambda_{k}^{\mathrm{W}}=k^{2} \pi^{2}$ (time is expressed in units of $\tau=L^{2} / D$ ), and the eigenvectors read $[67,72]$

$$
L_{k}^{\mathrm{W}}(x)=R_{k}^{\mathrm{W}}(x)=\delta_{k 0}+\left(1-\delta_{k 0}\right) \sqrt{2} \cos (k \pi x),
$$

since $\partial_{x}^{2}$ is self-adjoint. The mean local-time fraction, $\left\langle\theta_{x}(t)\right\rangle$, the variance $\sigma_{\theta}^{2}(t)$, and the covariance $C_{\theta_{1} \theta_{2}}(t)$ for the confined Wiener process are shown in Fig. 3. In the case of equilibrium initial conditions, $\left\langle\theta_{x}(t)\right\rangle_{\text {inv }}$ is constant and equal to $P_{\text {inv }}(x)$, and the fluctuations of $\theta_{x}(t)$ are largest at the boundaries as a result of repeated collisions with the walls. Notably, starting from localized conditions, $\left\langle\theta_{x}(t)\right\rangle_{x_{0}}$ as a function of $x$, in contrast to the ensemble propagator $P_{t}^{\mathrm{W}}\left(x \mid x_{0}\right)$, displays a persistent cusp located at the initial condition $x_{0}$ [see Fig. 3(c)]. The fluctuations of $\theta_{x}(t)$ are larger near the initial condition and at the boundaries.

\section{B. Local time fraction of the Ornstein-Uhlenbeck process}

Trajectories of the one-dimensional Ornstein-Uhlenbeck process are solutions of the Itô equation

$$
d x_{t}=-\gamma x_{t}+\sqrt{2 D} d W_{t}
$$

and on the level or probability density they correspond to the Fokker-Planck equation $\left(\partial_{t}-D\left[\partial_{x}^{2}+\gamma \partial_{x} x\right]\right) P_{t}^{\mathrm{OU}}\left(x \mid x_{0}\right)=$ 0 with initial condition $P_{0}^{\mathrm{OU}}\left(x \mid x_{0}\right)=\delta\left(x-x_{0}\right)$ and natural boundary conditions $\lim _{|x| \rightarrow \infty} P_{t}^{\mathrm{OU}}\left(x \mid x_{0}\right)=0$. To connect continuous processes to discrete ones, we translate the Fokker-Planck equation of the Ornstein-Uhlenbeck process to a random walk on a lattice with spacing $\Delta x$ and the harmonic potential $\gamma x^{2}$ entering transition rates according 

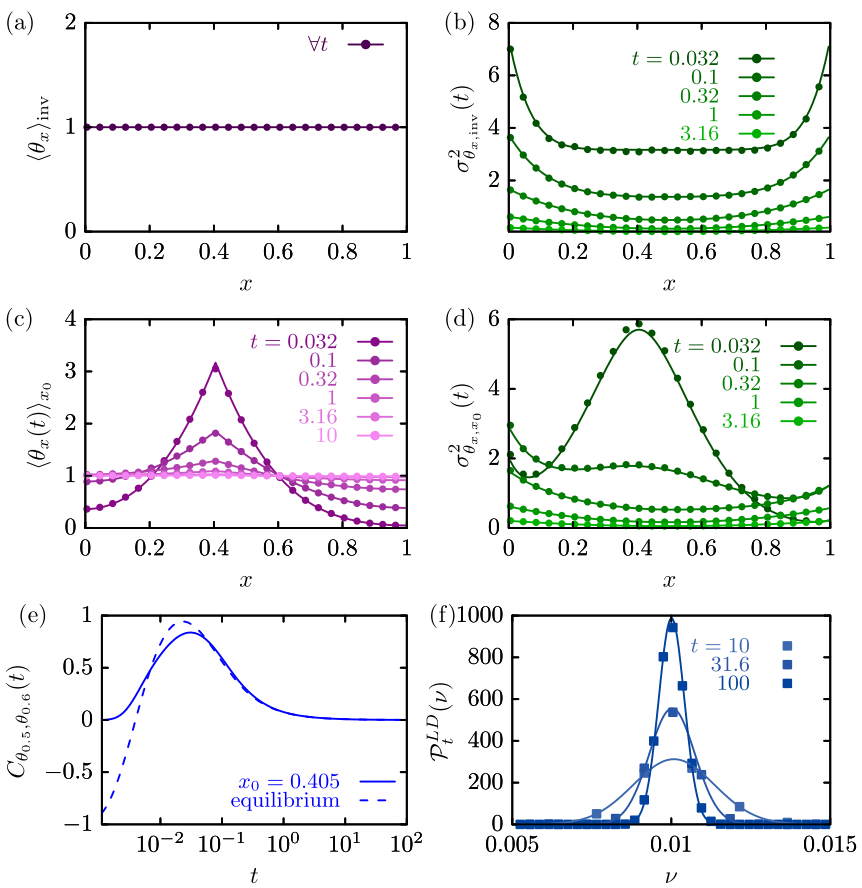

FIG. 3. Statistics of the fraction of local-time $\theta_{x}(t)$ as a function of $x$ at different times $t$ for equilibrium initial conditions, $p_{0}\left(x_{0}\right)=P_{\text {inv }}\left(x_{0}\right)[(\mathrm{a})$ and (b)] and localized initial condition at $x_{0}=0.405\left[\right.$ (c) and (d)]; (a) $\left\langle\theta_{x}(t)\right\rangle_{\text {inv }}$ is constant for all times; (b) $\sigma_{\theta_{x} \text {,inv }}^{2}(t)$ as a function of $x$ for equilibrium initial conditions; (c) $\left\langle\theta_{x}(t)\right\rangle_{x_{0}}$ and (d) $\sigma_{\theta_{x}}^{2}(t)$; (e) covariance starting from equilibrated initial conditions, $C_{\theta_{x} \theta_{y}}^{\text {inv }}(t)$ (dashed line) and localized initial conditions, $C_{\theta_{x} \theta_{y}}(t)$ (solid line). (f) Probability density of occupation time fraction $\theta_{\mathcal{V}}(t)=t^{-1} \int_{0}^{t} \mathbb{1}_{[0.45,0.55]}\left(x_{\tau}\right) d \tau$ [see also Eq. (7)] on large-deviation timescales (symbols) and corresponding theoretical result (56) (lines). Symbols were obtained from Brownian dynamics simulations of $10^{5}$ trajectories simulated with a time-step $d t=10^{-4}$.

to [80]

$$
\begin{aligned}
\langle x+\Delta x|\hat{L}| x\rangle & =\frac{D}{\Delta x^{2}} e^{\frac{1}{4} \gamma\left[x^{2}-(x+\Delta x)^{2}\right]}, \\
\langle x|\hat{L}| x+\Delta x\rangle & =\frac{D}{\Delta x^{2}} e^{\frac{1}{4} \gamma\left[(x+\Delta x)^{2}-x^{2}\right]},
\end{aligned}
$$

in a confined domain $\Omega_{\text {conf }}=\{-l,-l+\Delta x, \ldots, l-$ $\Delta x, l\} \subset \Omega$. The matrix $\hat{L}$ is tridiagonal and satisfies $\sum_{y}\langle y|\hat{L}| x\rangle=0$ for all $\mathbf{x} \in \Omega$ and $x \in \mathbb{Z} \Delta x$. We diagonalized $\hat{L}$ numerically using the library from Ref. [81]. The mean, variance, and correlation function for the continuous-space Ornstein-Uhlenbeck process (66) obtained from Brownian dynamics simulations are depicted in Fig. 4 (symbols) and are in excellent agreement with the spectral-theoretic results for the corresponding lattice random-walk approximation (67) (lines). In Fig. 4(f) we also investigate the full probability density function of the fraction of occupation time in the interval $x \in[0,0.01]$, i.e., $\theta_{\mathcal{V}}(t)=t^{-1} \int_{0}^{t} \mathbb{1}_{[0,0.01]}[x(\tau)] d \tau$ [see Eq. (7)] on large-deviation timescales, and we compare it to the theoretical Gaussian prediction Eq. (51). Note that while the eigenspectrum of the generator of continuous Ornstein-Uhlenbeck dynamics is unbounded, implying that the spectral-theoretic result would require the summation
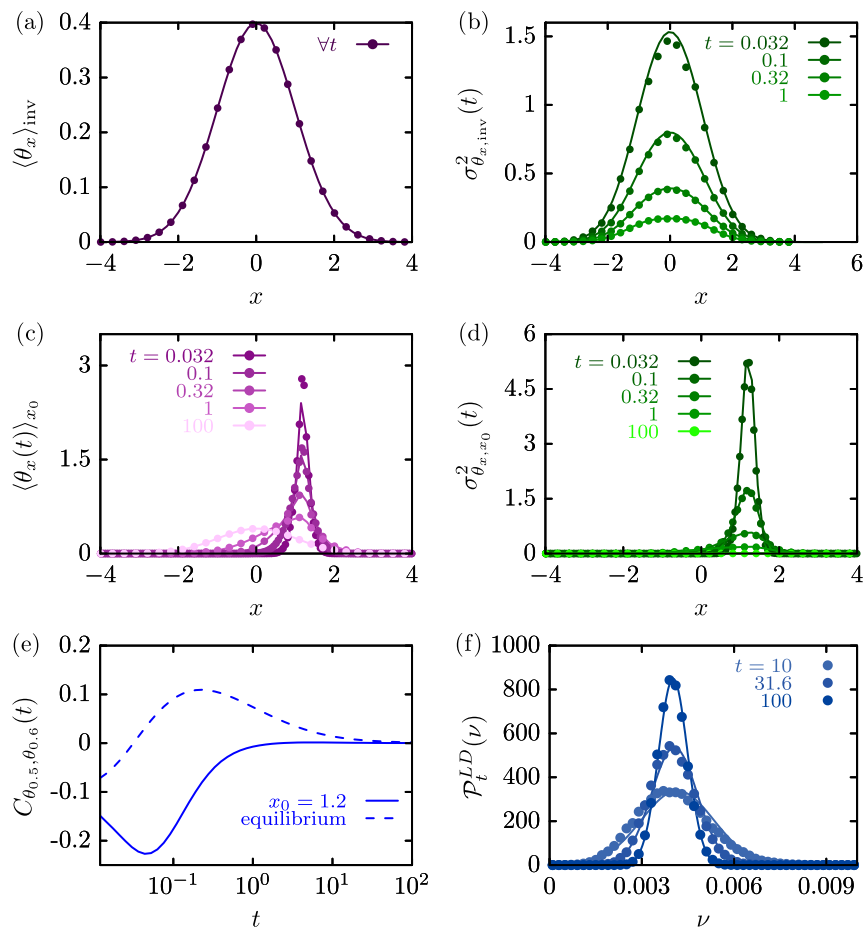

FIG. 4. Statistics of the fraction of local-time $\theta_{x}(t)$ as a function of $x$ for the Ornstein-Uhlenbeck process (symbols) and corresponding lattice random walk (67) with $10^{3}$ states in the interval $x \in$ $[-5,5]$ as a function of $x$ at different times $t$ for equilibrium initial conditions, i.e., $p_{0}\left(x_{0}\right)=P_{\text {inv }}\left(x_{0}\right)[(\mathrm{a})$ and (b)], and initial conditions localized at $x_{0}=1.2$, i.e., $p_{0}\left(x_{0}\right)=\delta\left(x_{0}-1.2\right)$ [(c) and (d)]. (a) $\left\langle\theta_{x}(t)\right\rangle_{\text {inv }}=P_{\text {inv }}(x)$ is a time-independent Gaussian; (b) $\sigma_{\theta_{x} \text {,inv }}^{2}(t)$ at various times as a function of $x$; (c) $\left\langle\theta_{x}(t)\right\rangle_{x_{0}}$ and (d) $\sigma_{\theta_{x}, x_{0}}^{2}(t)$ at various times as a function of $x$; (e) $C_{\theta_{0.5} \theta_{0.6}}(t)$ for equilibrium (dashed line) and localized (full lines) initial conditions; (f) occupation time fraction $\theta_{\mathcal{V}}(t)=t^{-1} \int_{0}^{t} \mathbb{1}_{[0,0.01]}[x(\tau)] d \tau$ [see also Eq. (7)] with symbols derived from simulations and the solid line representing the theoretical result (56) for the lattice random walk (67). To obtain each simulation point, we generated $10^{5}$ Brownian dynamics trajectories using $D=\gamma=1$ with a time-step $d t=10^{-4}$.

of a large number of terms, the summation in the lattice approximation is limited by the number of lattice points. Therefore, except for very short times, where the lattice approximation naturally breaks down, this example demonstrates that our formalism applies equally well to Markov-jump processes and diffusion dynamics. Note that the results in Figs. 4(c) and 4(d) for times $t=1$ and 100 correspond to the "short" and "long" trajectory in Fig. 2, respectively.

\section{Local-time fraction in a driven unicyclic network}

Let us address in the following a simple three-state model with broken detailed balance to also address driven systems. The model corresponds to a simple cycle with states 1,2 , and 3 , where all rates in a given direction are equal but each of them has the same forward/backward asymmetry. The model may represent, for example, a molecular motor such as the F1-ATPase driven by ATP hydrolysis [82]. The corresponding 

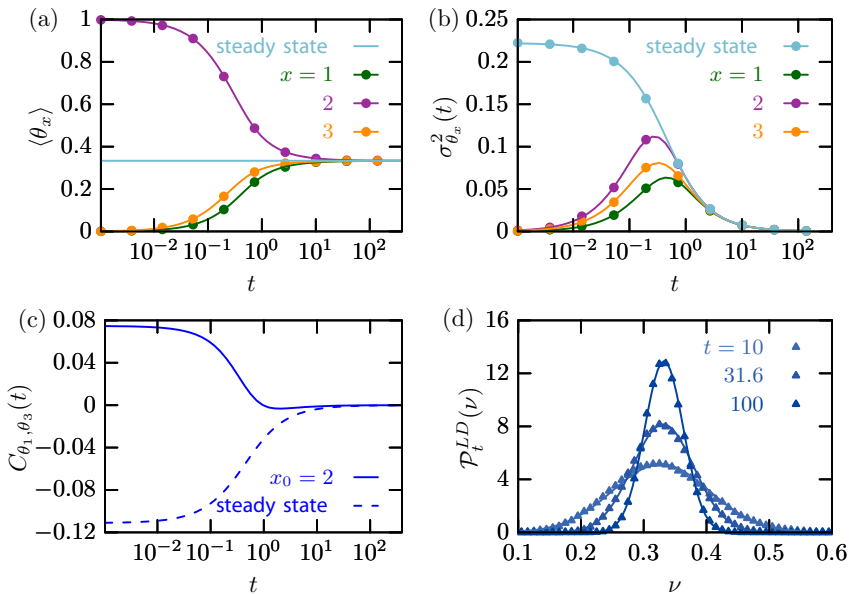

FIG. 5. (a) Mean local-time fraction $\left\langle\theta_{x}(t)\right\rangle$ in states $x=1,2,3$, respectively, for a driven unicyclic network starting from steady-state initial conditions $\left[p_{0}\left(x_{0}\right)=P_{\text {inv }}\left(x_{0}\right)\right.$, light blue line; result identical for any state] and an initial condition localized at $x_{0}=2$ (lines are theory and points simulations); (b) variance of the local-time fraction $\sigma_{\theta \text {,inv }}^{2}(t)$ starting from steady-state initial conditions (light blue line; result identical for any state) and $\sigma_{\theta, 2}^{2}(t)$ in states $x=1,2,3$ starting from $x_{0}=2$; (c) covariance of local-time fraction between states $x=1$ and $2, C_{\theta_{1} \theta_{2}}(t)$, as a function of time for stationary (dashed line) and localized (full line) initial conditions; (d) probability density function of the local-time fraction in state $x=1, \bar{V}_{t} \equiv$ $\theta_{1}(t)$, on large-deviation timescales alongside theoretical prediction of Eq. (56).

transition matrix of the model reads

$$
\hat{L}=\left(\begin{array}{rrr}
-3 & 1 & 2 \\
2 & -3 & 1 \\
1 & 2 & -3
\end{array}\right)
$$

and it has eigenvalues $\lambda_{0}=0, \lambda_{1,2}=-9 / 2 \pm i \sqrt{3} / 2$, and eigenvectors $\left|R_{0}\right\rangle=3^{-1}(1,1,1)^{T}$ and

$$
\left|R_{1,2}\right\rangle=\frac{1}{3}\left(\frac{-3 \sqrt{3} \pm i}{\sqrt{3} \mp 5 i}, \frac{2 \sqrt{3} \pm 2 i}{\sqrt{3} \mp 5 i}, 1\right)^{T} .
$$

As a result of broken detailed balance, the eigenspectrum is complex. In Fig. 5 we analyze the mean [panel (a)], fluctuations [panel (b)], and correlation function [panel (c)] of the local-time fraction $\theta_{x}(t)$ in the various states for nonequilibrium steady-state initial conditions (light blue lines) and conditions initially localized in state $x_{0}=2$, i.e., $\left|p_{0}\right\rangle=$ $(0,1,0)^{T}$. The theoretical results (lines) show an excellent agreement with simulations (symbols) carried out using the Gillespie algorithm [68]. We also confirm the Gaussian statistics of the local-time fraction $\theta_{x}(t)$ from Eq. (56) in Fig. 5(d).

\section{Generic behavior of the local-time fraction in ergodic systems}

Note that an exhaustive study of the statistics of the localtime fraction is beyond the scope of this work. Nevertheless, we discuss here some general features of $\theta_{x}(t)$. The manner in which $\left\langle\theta_{x}(t)\right\rangle$, starting from some nonequilibrium initial condition, approaches the ergodic invariant measure $P_{\text {inv }}(x)$ can be highly nontrivial and even nonmonotonic [see, e.g., Figs. 6(a)]. Even when the ergodic limit is reached, where
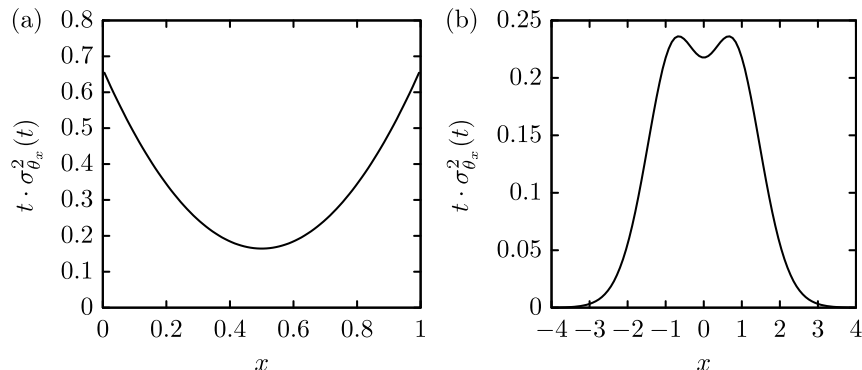

FIG. 6. Long-time behavior (i.e., $t \gg \lambda_{1}^{-1}$ ) of the scaled variance of the local-time, $t \sigma_{\theta_{x}}^{2}(t)$, that is time-independent; (a) results for the Wiener process and (b) the Ornstein-Uhlenbeck processes.

the variance ceases to depend on time, i.e., $t \sigma_{\theta_{x}}^{2}(t) \neq f(t)$, the fluctuations display a nontrivial behavior (see Fig. 6). For example, in the case of the Wiener process, fluctuations are enhanced close to the boundaries, while for the OrnsteinUhlenbeck process they become depressed near the minimum. Both results may be interpreted in terms of random "oscillations" around a typical position and confined by a boundary that amplifies fluctuations.

Moreover, the time dependence of $\left\langle\theta_{x}(t)\right\rangle$ for nonstationary initial conditions is often nonmonotonic or has a nonmonotonic derivative [see Figs. 7(a) and 7(c)]. A comparison between $\left\langle\theta_{x}(t)\right\rangle$ starting from stationary (dashed lines) and localized (full lines) initial conditions illustrates the two coexisting decorrelation mechanisms of $\theta_{x}(t)$ at different times, one corresponding to self-averaging and the emergence of the central-limit theorem (compare dashed and dotted lines), the other additionally reflecting the loss of memory of the initial condition (full lines). Stationary initial conditions often give rise to larger fluctuations than nonstationary initial conditions
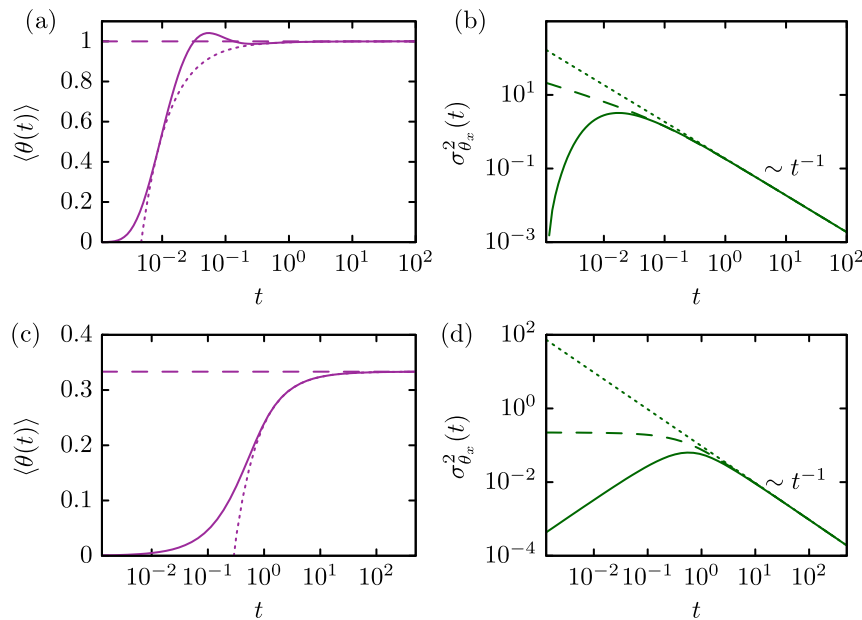

FIG. 7. (a) Mean local-time and (b) variance at $x=0.6$ for a Wiener process starting from equilibrium (dashed line) and from a localized initial condition $p_{0}(x)=\delta(x-0.405)$ (solid line). (a) Mean local-time $\left\langle\theta_{x}(t)\right\rangle$ and (b) variance $\sigma_{\theta_{x}}^{2}(t)$ at $x=0.6$ for the driven three-state cycle Eq. (68) starting from stationary (dashed line) and from a localized initial condition $p_{0}(x)=\delta(x-1)$ (solid line); the dotted lines correspond to the large-deviation limit (51) and depict the validity and long-time saturation of the upper bound Eq. (61). 
[compare the dashed and full lines in Figs. 7(b) and 7(d)], and in the particular case of equilibrium initial conditions for systems obeying detailed balance, $\sigma_{\theta}^{2}(t)$ is a monotonically decaying function of time $t$ [see Eq. (52) and Figs. 7(b) and 7(d)] with an upper bound given by the large-deviation asymptotic [see Eq. (61)] with a $\propto 1 / t$ scaling dictated by the central-limit theorem [dotted lines in Figs. 7(b) and 7(d)].

The covariance of the local-time fraction between a pair of points $x_{1}$ and $x_{2}$ in the continuous setting [Figs. 3(e) and 4(e)] or $x=1$ and 2 in the discrete setting [Fig. 5(c)], $C_{\theta_{1} \theta_{2}}(t)$, displays a similarly nontrivial and nonmonotonic dependence on time and initial conditions $p_{0}\left(x_{0}\right)$ as shown in Figs. 3(e), 4(e), and 5(c). The striking dependence on the tagged points reflects a directional persistence of individual trajectories in-between said points and can therefore be used as a robust indicator of directional persistence and thus "temporally correlated exploration" on the level of a single trajectory.

\section{Universal asymptotic Gaussian limit law for time-average physical observables}

Finally, we comment on the universal asymptotic Gaussian limit law Eq. (56) for Markovian as well as non-Markovian time-average physical observables of type (3) of ergodic stochastic dynamics of the form given in Eqs. (14) and (26). Namely, using the asymptotic results (47), (51), and (55) in the large-deviation probability density function (56), and rescaling to the centered and time-independent variables $\Xi$ and $\Xi_{i \mid j}$ defined in Eqs. (57) and (58), we can rescale the probability density of any time-average physical observable $\mathcal{P}_{t}^{\mathrm{LD}}(\hat{v})$, and the conditional probability density of a time-average physical observable given another time-average physical observable $\mathcal{P}_{t}^{\mathrm{LD}}\left(\hat{v}_{i} \mid \hat{v}_{j}\right)$, to collapse at long times onto a unit normal probability density (59). For the three models studied here, Figs. 3(f), 4(f), and 5(d), and additionally for the conditional probability density function of the occupation time fraction in $x \in[0.1,0.4]$ given the occupation time fraction in $y \in$ $[0.6,0.9]$ for the Wiener process, we demonstrate this collapse explicitly in Fig. 8.

\section{Precision limit of concentration measurement by a single receptor}

Let us now investigate the physical limit to the precision of concentration measurements by means of the simplest twostate Markov-jump process with states $\Omega=\{0,1\}$ [28]. The receptor can either be occupied by a ligand $(x=1)$ or be empty $(x=0)$. Let the background ligand concentration be $c$ and assume that the ligand binds with a rate $k c$ and unbinds with rate $k$, ignoring for simplicity any spatial variations of concentration. The generator and its eigenvectors are given by

$\hat{L}=\left(\begin{array}{cc}-k c & k \\ k c & -k\end{array}\right),\left|R_{0}\right\rangle=\frac{1}{1+c}\left(\begin{array}{l}1 \\ c\end{array}\right),\left|R_{1}\right\rangle=\frac{1}{1+c}\left(\begin{array}{c}1 \\ -1\end{array}\right)$

with $\lambda_{1}=-k(1+c)$ being the only nonzero eigenvalue. The left eigenvectors corresponding to Eq. (70) are $\left\langle L_{0}\right|=\langle-|=$ $(1,1)$ and $\left\langle L_{1}\right|=(c,-1)$. Moreover, since the entire state space has only two states, we have $\theta_{0}(t)=1-\theta_{1}(t)$. Assuming that the system was initially in equilibrium, $\left|p_{0}\right\rangle=\left|R_{0}\right\rangle$,

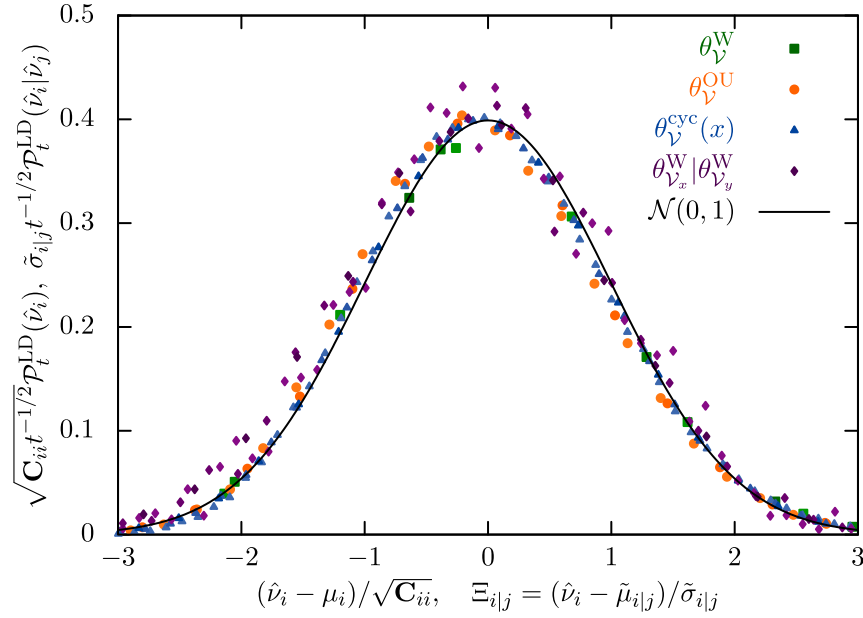

FIG. 8. Collapse of probability density functions of all studied models at long time onto a unit normal probability density. The symbols correspond to rescaled probability density of occupation time fraction $\theta_{\mathcal{V}}(t)^{\mathrm{W}, \mathrm{OU}, \mathrm{cyc}}$ [see also Eq. (7)] of the Wiener process (64), the Ornstein-Uhlenbeck process (66), the driven three-state cycle (68), respectively, and the conditional probability density function of occupation time fraction $\theta_{\mathcal{V}_{x}}(t)^{\mathrm{W}} \mid \theta_{\mathcal{V}_{y}}(t)^{\mathrm{W}}$ of the Wiener process for $\mathcal{V}_{x}=x \in[0.1,0.4]$ and $\mathcal{V}_{y}=y \in[0.6,0.9]$. The line is a zero mean unit normal probability density function $\mathcal{N}(0,1)$.

this implies that the mean values of the respective localtime fractions are given by $\left\langle\theta_{1}(t)\right\rangle=(1+c)^{-1} c$ and $\left\langle\theta_{0}(t)\right\rangle=$ $(1+c)^{-1}$.

If the receptor estimates the concentration $c$ by reading out and averaging the fraction of time the ligand is bound, $\theta_{1}$, over an interval of duration $t$, the precision of the estimate is bounded from above by the variance of the local-time fraction given by Eq. (52) and reads explicitly

$$
\sigma_{\theta_{1}}^{2}(t)=\frac{2 c}{(1+c)^{3} k t}\left[1-\frac{1-e^{-k(1+c) t}}{k(1+c) t}\right] .
$$

Typically one assumes that the measurement $t$ is longer than any correlation time $[28,30,83,84]$, which in the present setting implies $t \gg 1 /[k(1+c)]$, i.e., much longer than the correlation time of two-state Markov switching noise, $\tau_{c} \equiv$ $\lambda_{1}^{-1}=1 /(k+k c)[30]$. In this regime, the averaging noise corresponds to shot noise such that the variance decreases with the number of statistically independent receptor measurements $\#_{t}[28,30,83,84]$, where $\#_{t} \sim t / \tau_{c}$ is the number of statistically independent realizations of the two-state process. Therefore, $\sigma_{\theta_{1}}^{2}(t) \propto 1 / \#_{t}=\tau_{c} / t$, according to the centrallimit theorem.

Based on the bound derived in Eq. (61), the shot-noise limit is in fact an upper bound to fluctuations of receptor occupancy at any duration of measurement, and it saturates only in the limit $t \gg \tau_{c}$. Namely, a direct application of the bound (61) indeed yields, using $G_{t}^{\mathrm{eq}}(1,1)=P_{t}(1 \mid 1) P_{\infty}(1 \mid 1)$,

$$
\begin{aligned}
B & \equiv 2 \int_{0}^{\infty}\left[G_{t}^{\mathrm{eq}}(1,1)-G_{\infty}^{\mathrm{eq}}(1,1)\right] d t \\
& =2 \int_{0}^{\infty} \frac{c}{(1+c)^{2}} e^{-k(1+c) t} d t=\frac{2 c}{(1+c)^{3} k},
\end{aligned}
$$


implying, according to Eq. (61),

$$
\sigma_{\theta_{1}}^{2}(t) \leqslant \frac{B}{t}=\frac{2 c}{(1+c)^{2}} \frac{1}{\#_{t}} \equiv \lim _{t \gg \tau_{c}} \sigma_{\theta_{1}}^{2}(t) .
$$

Therefore, for short and particularly finite measurements, the shot-noise limit of fluctuations for long receptor read-out $[28,30,83,84]$ gives only an upper bound to the uncertainty of the estimate, whereas the inequality becomes sharp at long times.

Fundamental bounds on the precision of inferring $c$ from $\theta_{1}(t)$ can be found in [28]. Using the entire time trace of the receptor occupancy $x_{\tau}(0 \leqslant \tau \leqslant t)$ instead of the occupation time $\theta_{1}(t)$ alone, and employing a maximum-likelihood estimate of the concentration $c$, the error of the resulting estimate (i.e., its variance) is found to be reduced further by a factor of $1 / 2$ [31,32]. A detailed discussion of the precision of inferring kinetic parameters by means of nonlocal functionals can be found in Ref. [37].

\section{CONCLUDING PERSPECTIVE}

We developed a general spectral-theoretic approach to time-average statistical mechanics, i.e., to the statistics of bounded, local additive functionals of (normal) ergodic stochastic processes with continuous and discrete statespaces. In particular, we have shown how to obtain exactly the mean, variance, and correlations of time-average observables from the eigenspectrum of the underlying forward or backward generator. We rederived the famous Feynman-Kac formulas using Itô calculus and included a brief derivation for Markov-jump processes. We combined Feynman-Kac formulas with non-Hermitian perturbation theory to derive an exact spectral representation of the results. We demonstrated explicitly, and quantitatively, the emergence of the universal central-limit law in a spectral representation on large-deviation timescales. For the special case of equilibrated initial conditions and dynamics obeying detailed balance, we derived a general upper bound on fluctuations of occupation measures inferred from individual trajectories. We discussed our theoretical results from a physical perspective and provided simple but instructive practical examples to demonstrate how the theory is to be applied. Our work is applicable to continuous as well as discrete state-space processes, reversible as well as irreversible, encompassing a wide and diverse range of phenomena involving time-average observables and additive functionals in physical, chemical, and biological systems as well as financial mathematics and econophysics.

\section{ACKNOWLEDGMENTS}

The financial support from the German Research Foundation (DFG) through the Emmy Noether Program GO 2762/1-1 to A.G. is gratefully acknowledged.

\section{APPENDIX A: THE PERTURBATIVE CALCULATION}

We carry out all calculations with the spectrum of the backward generator $\hat{L}^{\dagger}$. Equivalent results can be derived using the forward generator instead. We carry out perturbative calculations (34) up to second order to derive the results in Eq. (37).

\section{Terms of first order in $u$}

Starting with a perturbation of the backward "kets" and collecting terms of first order in Eq. (34), we find

$$
-\hat{L}^{\dagger}\left|L_{k}^{1}\right\rangle+V\left|L_{k}\right\rangle=\lambda_{k}^{(1)}\left|L_{k}\right\rangle+\lambda_{k}^{\dagger}\left|L_{k}^{1}\right\rangle
$$

and multiply Eq. (A1) by $\left\langle R_{l}\right|$ from the left to obtain

$$
-\left\langle R_{l}\left|\hat{L}^{\dagger}\right| L_{k}^{1}\right\rangle+\left\langle R_{l}|V| L_{k}\right\rangle=\lambda_{k}^{(1)} \delta_{k l}+\lambda_{k}^{\dagger}\left\langle R_{l} \mid L_{k}^{1}\right\rangle .
$$

Therefore, if $k=l$ we find

$$
\lambda_{k}^{(1)}=\left\langle R_{k}|V| L_{k}\right\rangle \equiv V_{k k}
$$

while for $k \neq l$ we obtain

$$
\left\langle R_{l} \mid L_{k}^{1}\right\rangle=\frac{V_{l k}}{\lambda_{k}^{\dagger}-\lambda_{l}^{\dagger}}
$$

and therefore

$$
\left|L_{k}^{1}\right\rangle=\sum_{l \neq k} \frac{V_{l k}}{\lambda_{k}^{\dagger}-\lambda_{l}^{\dagger}}\left|L_{l}\right\rangle .
$$

We now turn to the perturbation of $\hat{L}^{\dagger}$ acting on the bras from the right and multiply the resulting first-order equation by $\left|L_{l}\right\rangle$ from the right to obtain

$$
\left.-\left\langle R_{k}^{1}\left|\hat{L}^{\dagger}\right| L_{l}\right\rangle+\left\langle R_{k}|V| L_{l}\right\rangle=\lambda_{k}^{\dagger}\left|R_{k}^{1}\right| L_{l}\right\rangle+\lambda_{k}^{(1)} \delta_{k l} .
$$

For $k=l$ we obtain the eigenvalue-corrections (A3) while for $k \neq l$ we have

$$
\left\langle R_{k}^{1}\right|=\sum_{l \neq k} \frac{V_{k l}}{\lambda_{k}^{\dagger}-\lambda_{l}^{\dagger}}\left\langle R_{l}\right| .
$$

\section{Terms of second order in $u$}

Collecting in Eq. (34) corrections of second order to the kets we find, upon multiplying by $\left\langle R_{l}\right|$ from the left,

$$
\begin{aligned}
& -\left\langle R_{l}\left|\hat{L}^{\dagger}\right| L_{k}^{2}\right\rangle+\left\langle R_{l}|V| L_{k}^{1}\right\rangle \\
& \left.\left.\quad=\lambda_{k}^{(2)} \delta_{l k}+\lambda_{k}^{(1)}\left|R_{l}\right| L_{k}^{1}\right\rangle+\lambda_{k}^{\dagger}\left|R_{l}\right| L_{k}^{2}\right\rangle,
\end{aligned}
$$

yielding, for $k=l$,

$$
\lambda_{k}^{(2)}=\left\langle R_{k}^{0}|\hat{V}| L_{k}^{1}\right\rangle,
$$

because $\left\langle R_{k} \mid L_{k}^{1}\right\rangle=0$ due to the Eq. (36) and thus

$$
\lambda_{k}^{(2)}=\sum_{l \neq k} \frac{V_{k l} V_{l k}}{\lambda_{k}^{\dagger}-\lambda_{l}^{\dagger}}\left\langle R_{l}|V| L_{k}\right\rangle .
$$

Conversely, if $k \neq l$ we obtain the second-order correction

$$
\left|L_{k}^{2}\right\rangle=\sum_{l \neq k}\left[\sum_{i \neq k} \frac{V_{i k} V_{l i}}{\left(\lambda_{i}^{\dagger}-\lambda_{k}^{\dagger}\right)\left(\lambda_{l}^{\dagger}-\lambda_{k}^{\dagger}\right)}-\frac{V_{k k} V_{l k}}{\left(\lambda_{l}^{\dagger}-\lambda_{k}^{\dagger}\right)^{2}}\right]\left|L_{l}\right\rangle .
$$

Collecting in Eq. (34) corrections of second order to the bras we find, upon multiplying from the right by $\left|L_{l}\right\rangle$,

$$
\begin{aligned}
& -\left\langle R_{k}^{2}\left|\hat{L}^{\dagger}\right| L_{l}\right\rangle+\left\langle R_{k}^{1}|V| L_{l}\right\rangle \\
& \left.=\lambda_{k}^{(2)} \delta_{k l}+\lambda_{k}^{(1)}\left\langle R_{k}^{1} \mid L_{l}\right\rangle+\lambda_{k}^{\dagger}\left|R_{k}^{2}\right| L_{l}\right\rangle .
\end{aligned}
$$


When $k=l$ we obtain Eq. (A10) while in the case when $k \neq l$ we find the second correction to the bra,

$$
\left\langle R_{k}^{2}\right|=\sum_{l \neq k}\left[\sum_{i \neq k} \frac{V_{k i} V_{i l}}{\left(\lambda_{i}^{\dagger}-\lambda_{k}^{\dagger}\right)\left(\lambda_{l}^{\dagger}-\lambda_{k}^{\dagger}\right)}-\frac{V_{k k} V_{k l}}{\left(\lambda_{l}^{\dagger}-\lambda_{k}^{\dagger}\right)^{2}}\right]\left\langle R_{l}\right|
$$

which completes the derivation of Eq. (37).

\section{APPENDIX B: DERIVATION VIA THE DYSON SERIES}

In a previous publication [59], we showed how to derive equations for the moments of $\psi_{t}$ for stationary initial conditions, $p_{0}(\mathbf{x})=P_{\text {inv }}(\mathbf{x})$ [Eqs. (52) and (54)], using a Dyson series approach. Here we sketch how to obtain the moments of $\psi_{t}$ for generic initial conditions $p_{0}(\mathbf{x})$. In contrast to Ref. [59], we use the forward approach here and expand the tilted propagator up to the second order in $u$,

$$
\left\langle-\left|e^{(\hat{L}-u V) t}\right| p_{0}\right\rangle=1-u\left\langle-\left|\int_{0}^{t} d t^{\prime} e^{\hat{L}\left(t-t^{\prime}\right)} V e^{\hat{L} t^{\prime}}\right| p_{0}\right\rangle+u^{2}\left\langle-\left|\int_{0}^{t} d t^{\prime} \int_{0}^{t^{\prime}} d t^{\prime \prime} e^{\hat{L}\left(t-t^{\prime}\right)} V e^{\hat{L}\left(t^{\prime}-t^{\prime \prime}\right)} V e^{\hat{L} t^{\prime \prime}}\right| p_{0}\right\rangle+O\left(u^{3}\right),
$$

assuming $t>t^{\prime}>t^{\prime \prime}>0$. Here we confirm that the Dyson series gives results identical to the perturbation calculation.

\section{Mean, fluctuations, and correlations}

We now derive $\left\langle\bar{V}_{t}\right\rangle, \sigma_{\bar{V}}^{2}(t)$, and $C_{\bar{V}_{1} \bar{V}_{2}}(t)$ presented in Eqs. (46)-(54) using the Dyson series. Note that this calculation does not diagonalize the tilted generator $\hat{L}^{\dagger}-u V(\mathbf{x})\left[\hat{L}^{\dagger}-u V(\mathbf{x})\right.$, respectively]. Starting from Eq. (B1), we can carry out all integrations analytically for arbitrary initial conditions $p_{0}(\mathbf{x})$. To first order in $u$, we obtain

$$
\begin{aligned}
t^{-1}\left\langle-\left|\int_{0}^{t} d t^{\prime} e^{\hat{L}\left(t-t^{\prime}\right)} V e^{\hat{L} t^{\prime}}\right| p_{0}\right\rangle & =t^{-1} \int_{0}^{t} d t^{\prime} \sum_{k} \sum_{l}\left\langle-\mid R_{l}\right\rangle\left\langle L_{l}|V| R_{k}\right\rangle\left\langle L_{k} \mid p_{0}\right\rangle e^{-\lambda_{l}\left(t-t^{\prime}\right)-\lambda_{k} t^{\prime}} \\
& =t^{-1} \int_{0}^{t} d t^{\prime} \sum_{k}\left\langle-|V| R_{k}\right\rangle\left\langle L_{k} \mid p_{0}\right\rangle e^{-\lambda_{k} t^{\prime}}=V_{00}+\frac{1}{t} \sum_{k} \frac{V_{k 0}}{\lambda_{k}}\left\langle L_{k} \mid p_{0}\right\rangle\left(1-e^{-\lambda_{k} t}\right),
\end{aligned}
$$

where for $p_{0}(\mathbf{x})=P_{\text {inv }}(\mathbf{x})$ we have $\left\langle L_{k} \mid p_{0}\right\rangle=\delta_{k 0}$ leading to $\langle\bar{V}\rangle_{\text {inv }}=V_{00}=P_{\text {inv }}(\mathbf{x})$.

To second order in $u$ we find for an arbitrary $p_{0}(\mathbf{x})$

$$
\begin{aligned}
& t^{-2}\left\langle-\left|\int_{0}^{t} d t^{\prime} \int_{0}^{t^{\prime}} d t^{\prime \prime} e^{\hat{L}\left(t-t^{\prime}\right)} V e^{\hat{L}\left(t^{\prime}-t^{\prime \prime}\right)} V e^{\hat{L} t^{\prime \prime}}\right| p_{0}\right\rangle \\
& =t^{-2} \int_{0}^{t} d t^{\prime} \int_{0}^{t^{\prime}} d t^{\prime \prime}\left\langle-\left|\sum_{m}\right| R_{m}\right\rangle\left\langle L_{m}\left|e^{-\lambda_{m}\left(t-t^{\prime}\right)} V \sum_{k}\right| R_{k}\right\rangle\left\langle L_{k}\left|e^{-\lambda_{k}\left(t^{\prime}-t^{\prime \prime}\right)} V \sum_{l}\right| R_{i}\right\rangle\left\langle L_{l}\left|e^{-\lambda_{l} t^{\prime \prime}}\right| p_{0}\right\rangle \\
& =t^{-2} \sum_{k} \sum_{l} V_{0 k} V_{k l}\left\langle L_{l} \mid p_{0}\right\rangle \int_{0}^{t} d t^{\prime} \int_{0}^{t^{\prime}} d t^{\prime \prime} e^{-\lambda_{k}\left(t^{\prime}-t^{\prime \prime}\right)-\lambda_{l} t^{\prime \prime}}
\end{aligned}
$$

when $k=l=0$ only $V_{00}^{2} / 2$ survives, while for $k \neq 0$ and $l=$ 0 we find

$$
\frac{1}{t} \sum_{k>0} \frac{V_{k 0} V_{0 k}}{\lambda_{k}}\left(1-\frac{1-e^{-\lambda_{k} t}}{t \lambda_{k}}\right)
$$

Conversely, when $k=0$ and $l \neq 0$ we end up with

$$
\frac{1}{t} \sum_{l>0} \frac{V_{00} V_{l 0}}{\lambda_{l}}\left\langle L_{l} \mid p_{0}\right\rangle\left(1-\frac{1-e^{-\lambda_{l} t}}{t \lambda_{l}}\right)
$$

while for $k=l$ and $k \neq 0$ and $l \neq 0$ we have

$$
\frac{1}{t} \sum_{k>0} \frac{V_{k 0} V_{k k}}{\lambda_{k}}\left\langle L_{k} \mid p_{0}\right\rangle\left(e^{-\lambda_{k} t}-\frac{1-e^{-\lambda_{k} t}}{t \lambda_{k}}\right) .
$$

Finally, when $k \neq l \neq 0$ we obtain

$$
\frac{1}{t^{2}} \sum_{k>0} \sum_{l>0, l \neq k} \frac{V_{k 0} V_{l k}}{\lambda_{k}-\lambda_{l}}\left\langle L_{l} \mid p_{0}\right\rangle\left(\frac{1-e^{-\lambda_{l} t}}{\lambda_{l}}-\frac{1-e^{-\lambda_{k} t}}{\lambda_{k}}\right) .
$$

The sum of these terms yields the sought-after result. The corresponding result for stationary initial conditions, $p_{0}(\mathbf{x})=$ $P_{\text {inv }}(\mathbf{x})$, is obtained using $\left\langle L_{l} \mid P_{\text {inv }}\right\rangle=\delta_{l 0}$, which leads to Eqs. (52) and (54). When considering correlations, we make the replacement $u V \rightarrow u_{1} V_{1}+u_{2} V_{2}$ and replace $\partial_{u_{1}}^{2} \rightarrow \partial_{u_{1} u_{2}}^{2}$ to compute the covariance. The formulas above thereby generalize in a straightforward manner.
[1] M. J. Saxton, Single-particle tracking: Connecting the dots, Nat. Methods 5, 671 (2008).
[2] R. Metzler, J.-H. Jeon, A. G. Cherstvy, and E. Barkai, Anomalous diffusion models and their properties: Non-stationarity, 
non-ergodicity, and ageing at the centenary of single particle tracking, Phys. Chem. Chem. Phys. 16, 24128 (2014).

[3] D. Ernst, J. Köhler, and M. Weiss, Probing the type of anomalous diffusion with single-particle tracking, Phys. Chem. Chem. Phys. 16, 7686 (2014).

[4] H. Shen, L. J. Tauzin, R. Baiyasi, W. Wang, N. Moringo, B. Shuang, and C. F. Landes, Single particle tracking: From theory to biophysical applications, Chem. Rev. 117, 7331 (2017).

[5] M. L. Hughes and L. Dougan, The physics of pulling polyproteins: a review of single molecule force spectroscopy using the AFM to study protein unfolding, Rep. Prog. Phys. 79, 076601 (2016).

[6] X. Sunney Xie, Single-molecule spectroscopy and dynamics at room temperature, Acc. Chem. Res. 29, 598 (1996).

[7] W. P. Ambrose, P. M. Goodwin, J. H. Jett, A. Van Orden, J. H. Werner, and R. A. Keller, Single molecule fluorescence spectroscopy at ambient temperature, Chem. Rev. 99, 2929 (1999).

[8] T. Plakhotnik, E. A. Donley, and U. P. Wild, Single-molecule spectroscopy, Annu. Rev. Phys. Chem. 48, 181 (1997).

[9] K. C. Neuman and A. Nagy, Single-molecule force spectroscopy: Optical tweezers, magnetic tweezers and atomic force microscopy, Nat. Methods 5, 491 (2008).

[10] M. Rief and H. Grubmüller, Force spectroscopy of single biomolecules, ChemPhysChem 3, 255 (2002).

[11] M. T. Woodside and S. M. Block, Reconstructing folding energy landscapes by single-molecule force spectroscopy, Annu. Rev. Biophys. 43, 19 (2014).

[12] F. Ritort, Single-molecule experiments in biological physics: methods and applications, J. Phys.: Condens. Matter 18, R531 (2006).

[13] J. Camunas-Soler, M. Ribezzi-Crivellari, and F. Ritort, Elastic properties of nucleic acids by single-molecule force spectroscopy, Annu. Rev. Biophys. 45, 65 (2016).

[14] P. Lévy, Sur certains processus stochastiques homogènes, Compositio Mathematica 7, 283 (1940).

[15] M. Kac, On distributions of certain Wiener functionals, Trans. Am. Math. Soc. 65, 1 (1949).

[16] D. A. Darling and M. Kac, On occupation times for Markoff processes, Trans. Am. Math. Soc. 84, 444 (1957).

[17] J. Lamperti, An occupation time theorem for a class of stochastic processes, Trans. Am. Math. Soc. 88, 380 (1958).

[18] W. Feller, Fluctuation theory of recurrent events, Trans. Am. Math. Soc. 67, 98 (1949).

[19] N. H. Bingham, Fluctuation theory in continuous time, Adv. Appl. Probab. 7, 705 (1975).

[20] A N Borodin, Brownian local time, Russ. Math. Surv. 44, 1 (1989).

[21] J.-Y. Yen and M. Yor, Local Times and Excursion Theory for Brownian Motion (Springer, Cham, 2013).

[22] M. Yor, Exponential Functionals of Brownian Motion and Related Processes, Springer Finance Lecture Notes (SpringerVerlag, Berlin, 2001).

[23] H. Geman and M. Yor, Bessel processes, Asian options, and perpetuities, Math. Fin. 3, 349 (1993).

[24] G. Wilemski and M. Fixman, General theory of diffusioncontrolled reactions, J. Chem. Phys. 58, 4009 (1973).

[25] A. Szabo, Theory of diffusion-influenced fluorescence quenching, J. Phys. Chem. 93, 6929 (1989).
[26] O. Bénichou, M. Coppey, M. Moreau, and G. Oshanin, Kinetics of diffusion-limited catalytically activated reactions: An extension of the Wilemski-Fixman approach, J. Chem. Phys. 123, 194506 (2005).

[27] D. S. Grebenkov, Residence times and other functionals of reflected Brownian motion, Phys. Rev. E 76, 041139 (2007).

[28] H. C. Berg and E. M. Purcell, Physics of chemoreception, Biophys. J. 20, 193 (1977).

[29] F. W. Wiegel, Diffusion and the physics of chemoreception, Phys. Rep. 95, 283 (1983).

[30] W. Bialek and S. Setayeshgar, Physical limits to biochemical signaling, Proc. Natl. Acad. Sci. (USA) 102, 10040 (2005).

[31] R. G. Endres and N. S. Wingreen, Maximum Likelihood and the Single Receptor, Phys. Rev. Lett. 103, 158101 (2009).

[32] T. Mora and N. S. Wingreen, Limits of Sensing Temporal Concentration Changes by Single Cells, Phys. Rev. Lett. 104, 248101 (2010).

[33] A. H. Lang, C. K. Fisher, T. Mora, and P. Mehta, Thermodynamics of Statistical Inference by Cells, Phys. Rev. Lett. 113, 148103 (2014).

[34] T. Mora, Physical Limit to Concentration Sensing Amid Spurious Ligands, Phys. Rev. Lett. 115, 038102 (2015).

[35] A. C. Barato and U. Seifert, Dispersion for two classes of random variables: General theory and application to inference of an external ligand concentration by a cell, Phys. Rev. E 92, 032127 (2015).

[36] G. Aquino, N. S. Wingreen, and R. G. Endres, Know the singlereceptor sensing limit? Think again, J. Stat. Phys. 162, 1353 (2015).

[37] D. Hartich and U. Seifert, Optimal inference strategies and their implications for the linear noise approximation, Phys. Rev. E 94, 042416 (2016).

[38] M. Ferraro and L. Zaninetti, Statistics of visits to sites in random walks, Physica A 338, 307 (2004).

[39] A. H. Gandjbakhche and G. H. Weiss, Descriptive parameter for photon trajectories in a turbid medium, Phys. Rev. E 61, 6958 (2000).

[40] G. H. Weiss and P. P. Calabrese, Occupation times of a CTRW on a lattice with anomalous sites, Physica A 234, 443 (1996).

[41] Z. Toroczkai, T. J. Newman, and S. Das Sarma, Sign-time distributions for interface growth, Phys. Rev. E 60, R1115 (1999).

[42] X. Brokmann, J.-P. Hermier, G. Messin, P. Desbiolles, J.-P. Bouchaud, and M. Dahan, Statistical Aging and Nonergodicity in the Fluorescence of Single Nanocrystals, Phys. Rev. Lett. 90, 120601 (2003).

[43] F. D. Stefani, J. P. Hoogenboom, and E. Barkai, Beyond quantum jumps: Blinking nanoscale light emitters, Phys. Today 62(2), 34 (2009).

[44] A. Comtet, J. Desbois, and C. Texier, Functionals of Brownian motion, localization and metric graphs, J. Phys. A 38, R341 (2005).

[45] S. N. Majumdar and A. J. Bray, Large-deviation functions for nonlinear functionals of a Gaussian stationary Markov process, Phys. Rev. E 65, 051112 (2002).

[46] S. N. Majumdar, Brownian functionals in physics and computer science, Curr. Sci. 89, 2075 (2005).

[47] S. Wennmalm, L. Edman, and R. Rigler, Conformational fluctuations in single DNA molecules, Proc. Natl. Acad. Sci. (USA) 94, 10641 (1997). 
[48] I. V. Gopich and A. Szabo, Theory of the energy transfer efficiency and fluorescence lifetime distribution in single-molecule FRET, Proc. Natl. Acad. Sci. (USA) 109, 7747 (2012).

[49] L. Fleury, J.-M. Segura, G. Zumofen, B. Hecht, and U. P. Wild, Nonclassical Photon Statistics in Single-Molecule Fluorescence at Room Temperature, Phys. Rev. Lett. 84, 1148 (2000).

[50] E. Barkai, Y. J. Jung, and R. Silbey, Theory of single-molecule spectroscopy: Beyond the ensemble average, Annu. Rev. Phys. Chem. 55, 457 (2004).

[51] N. Agmon, The residence time equation, Chem. Phys. Lett. 497, 184 (2010).

[52] S. Sabhapandit, S. N. Majumdar, and A. Comtet, Statistical properties of functionals of the paths of a particle diffusing in a one-dimensional random potential, Phys. Rev. E 73, 051102 (2006).

[53] S. N. Majumdar and A. Comtet, Local and Occupation Time of a Particle Diffusing in a Random Medium, Phys. Rev. Lett. 89, 060601 (2002).

[54] G. Bel and E. Barkai, Weak Ergodicity Breaking in the Continuous-Time Random Walk, Phys. Rev. Lett. 94, 240602 (2005).

[55] S. Carmi and E. Barkai, Fractional Feynman-Kac equation for weak ergodicity breaking, Phys. Rev. E 84, 061104 (2011).

[56] A. Dhar and S. N. Majumdar, Residence time distribution for a class of Gaussian Markov processes, Phys. Rev. E 59, 6413 (1999).

[57] S. N. Majumdar and D. S. Dean, Exact occupation time distribution in a non-Markovian sequence and its relation to spin glass models, Phys. Rev. E 66, 041102 (2002).

[58] A. J. Bray, S. N. Majumdar, and G. Schehr, Persistence and first-passage properties in nonequilibrium systems, Adv. Phys. 62, 225 (2013).

[59] A. Lapolla and A. Godec, Unfolding tagged particle histories in single-file diffusion: exact single- and two-tag local times beyond large deviation theory, New J. Phys. 20, 113021 (2018).

[60] A. Lapolla and A. Godec, Manifestations of projection-induced memory: General theory and the tilted single file, Front. Phys. 7, 182 (2019).

[61] D. Boyer, D. S. Dean, C. Mejía-Monasterio, and G. Oshanin, Optimal fits of diffusion constants from single-time data points of Brownian trajectories, Phys. Rev. E 86, 060101(R) (2012).

[62] D. Boyer, D. S. Dean, C. Mejía-Monasterio, and G. Oshanin, Optimal estimates of the diffusion coefficient of a single Brownian trajectory, Phys. Rev. E 85, 031136 (2012).

[63] J. B. Jørgensen, J. Mann, S. Ott, H. L. Pócseli, and J. Trulsen, Experimental studies of occupation and transit times in turbulent flows, Phys. Fluids 17, 035111 (2005).

[64] A. C. Barato and R. Chetrite, A formal view on level 2.5 large deviations and fluctuation relations, J. Stat. Phys. 160, 1154 (2015).
[65] H. Touchette, The large deviation approach to statistical mechanics, Phys. Rep. 478, 1 (2009).

[66] H. Touchette, Introduction to dynamical large deviations of markov processes, Physica A 504, 5 (2018).

[67] C. W. Gardiner, Handbook of Stochastic Methods, 3rd ed. (Springer, Berlin, 2004).

[68] D. T. Gillespie, Exact stochastic simulation of coupled chemical reactions, J. Phys. Chem. 81, 2340 (1977).

[69] J. Schnakenberg, Network theory of microscopic and macroscopic behavior of master equation systems, Rev. Mod. Phys. 48, 571 (1976).

[70] J. B. Conway, A Course in Functional Analysis, Graduate Texts in Mathematics (Springer-Verlag, New York, 1985).

[71] N. G. van Kampen, Stochastic Processes in Physics and Chemistry, 3rd ed., North-Holland Personal Library (Elsevier, Amsterdam, 2007).

[72] H. Risken, The Fokker-Planck Equation: Methods of Solution and Applications, 2nd ed., Springer Series in Synergetics (Springer-Verlag, Berlin, 1996).

[73] S. D. Stoller, W. Happer, and F. J. Dyson, Transverse spin relaxation in inhomogeneous magnetic fields, Phys. Rev. A 44, 7459 (1991).

[74] Note that 0 is an eigenvalue of $\hat{L}$ and $\tilde{\mathcal{P}}_{s}^{\psi}\left(\mathbf{u} \mid p_{0}\right)$ is meromorphic for small $|\mathbf{u}|$, therefore we can assume, without loss of generality, that $\mathbf{u}$ is real.

[75] J. Marcinkiewicz, Sur une propriété de la loi de Gauß, Math. Z. 44, 612 (1939).

[76] J. J. Sakurai and J. Napolitano, Modern Quantum Mechanics (Cambridge University Press, Cambridge, 2017).

[77] D. J. Klein, Degenerate perturbation theory, J. Chem. Phys. 61, 786 (1974).

[78] D. Hartich and A. Godec, Extreme value statistics of ergodic Markov processes from first passage times in the large deviation limit, J. Phys. A 52, 244001 (2019).

[79] A. I. Khinchin, Sur la loi des grands nombres, C. R. Acad. Sci. 188, 477 (1929).

[80] V. Holubec, K. Kroy, and S. Steffenoni, Physically consistent numerical solver for time-dependent Fokker-Planck equations, Phys. Rev. E 99, 032117 (2019).

[81] G. Guennebaud, B. Jacob et al., Eigen v3, http://eigen. tuxfamily.org (2010).

[82] S. Toyabe, T. Watanabe-Nakayama, T. Okamoto, S. Kudo, and E. Muneyuki, Thermodynamic efficiency and mechanochemical coupling of $\mathrm{F}_{1}$-ATPase, Proc. Natl. Acad. Sci. (USA) 108, 17951 (2011).

[83] A. Godec and R. Metzler, Signal focusing through active transport, Phys. Rev. E 92, 010701(R) (2015).

[84] A. Godec and R. Metzler, Active transport improves the precision of linear long distance molecular signalling, J. Phys. A 49, 364001 (2016). 